\title{
Beyond knowing that: A new generation of epistemic logics*
}

\author{
Yanjing Wang
}

\begin{abstract}
Epistemic logic has become a major field of philosophical logic ever since the groundbreaking work by Hintikka (1962). Despite its various successful applications in theoretical computer science, AI, and game theory, the technical development of the field has been mainly focusing on the propositional part, i.e., the propositional modal logics of "knowing that". However, knowledge is expressed in everyday life by using various other locutions such as "knowing whether", "knowing what", "knowing how" and so on (knowing-wh hereafter). Such knowledge expressions are better captured in quantified epistemic logic, as was already discussed by Hintikka (1962) and his sequel works at length. This paper aims to draw the attention back again to such a fascinating but largely neglected topic. We first survey what Hintikka and others did in the literature of quantified epistemic logic, and then advocate a new quantifier-free approach to study the epistemic logics of knowing-wh, which we believe can balance expressivity and complexity, and capture the essential reasoning patterns about knowing-wh. We survey our recent line of work on the epistemic logics of "knowing whether", "knowing what" and "knowing how" to demonstrate the use of this new approach.
\end{abstract}

\section{Introduction}

Epistemic logic as a field was created and largely shaped by Jaakko Hintikka's groundbreaking work. Starting from the very beginning, Hintikka (1962) set the stage of epistemic logic in favor of a possible world semantics 2 whose rich and

Yanjing Wang

Department of Philosophy, Peking University, e-mail: $y \cdot$ wang@pku • edu • cn

* A mindmap of this paper is here: http://www.phil.pku.edu.cn/personal/wangyj/mindmaps/

${ }^{2}$ Hintikka was never happy with the term "possible worlds", since in his models there may be no "worlds" but only situations or states, which are partial descriptions of the worlds. However, in this paper we will still use the term "possible worlds" for convenience. 
intuitive structure facilitates in-depth philosophical discussions and an intuitive understanding of knowledge that leads to various applications in other fields such as distributed systems and artificial intelligence. In a nutshell, Hintikka's notion of knowledge amounts to the elimination of uncertainty. At a given world, the alternative relation induces a split of all the possible worlds: the epistemically possible ones and the rest. The agent knows $\varphi$ at a world iff the $\neg \varphi$ worlds are ruled out in its epistemic alternatives according to the agent. In fact, such a semantics also works for other propositional attitudes that are essentially about information, such as belief (Hintikka, 2003).

Hintikka (1962) devoted most of the book on propositional epistemic logic with the following language (call it EL):

$$
\varphi::=\top|p| \neg \varphi|(\varphi \wedge \varphi)| K_{i} \varphi
$$

where $K_{i} \varphi$ reads "agent $i$ knows that $\varphi$ ". The language is interpreted on Kripke models $\mathscr{M}=\left\langle S,\left\{\rightarrow_{i} \mid i \in \mathbf{I}\right\}, V\right\rangle$ where $S$ is a non-empty set of possible worlds, $\rightarrow{ }_{i} \subseteq S \times S$ and $V: \mathbf{P} \rightarrow 2^{S}$. The semantics for $K_{i} \varphi$ is as follows:

$$
\mathscr{M}, s \vDash K_{i} \varphi \Leftrightarrow \text { for all } t \text { such that } s \rightarrow_{i} t: \mathscr{M}, t \vDash \varphi
$$

According to Hintikka (1962), $\rightarrow_{i}$ should be reflexive and transitive. In many applications, it is also reasonable to take it as an equivalence relation, which gives rise to the $\mathbb{S} 5$ axiom system, a very strong epistemic logic (Fagin et al, 1995):

$$
\begin{array}{lccc} 
& \multicolumn{1}{c}{\text { System } \mathbb{S} 5} & \multicolumn{2}{c}{\text { Rules }} \\
\text { Axioms } & \multicolumn{1}{c}{\begin{array}{l}
\varphi \rightarrow \psi \\
\text { TAUT }
\end{array}} & \text { all the instances of tautologies MP } & \frac{\varphi, \varphi}{\psi} \\
\text { DISTK } & K_{i}(p \rightarrow q) \rightarrow\left(K_{i} p \rightarrow K_{i} q\right) & \mathrm{NECK} & \frac{\varphi}{K_{i} \varphi} \\
\text { T } & K_{i} p \rightarrow p & \text { SUB } & \frac{\varphi}{\varphi[p / \psi]} \\
4 & K_{i} p \rightarrow K_{i} K_{i} p & & \\
5 & \neg K_{i} p \rightarrow K_{i} \neg K_{i} p & &
\end{array}
$$

Despite various philosophical debates regarding the axioms 4 and 5, and the problem of logical omniscience (cf. (Lenzen, 1978)), propositional epistemic logic has been successfully applied to many other fields because its semantic notion of knowledge is intuitive and flexible enough to handle uncertainties in various contexts. The knowledge modality $K_{i}$ is in particular powerful when combined with other modalities such as the temporal ones and the action modalities, which resulted in two influential approaches which can model changes of knowledge: Epistemic Temporal Logic (ETL) and Dynamic Epistemic Logic (DEL) (cf. e.g., Fagin et al (1995); van Ditmarsch et al (2007)). See van Ditmarsch et al (2015) for an overview of the applications of epistemic logic. 
However, knowledge is not only expressed by "knowing that". For example, we often use the verb "know" with an embedded question such as:

- I know whether the claim is true.

- I know what your password is.

- I know how to swim.
- I know why he was late.

- I know who proved this theorem.

- I know where she has been.

In the rest of the paper, we call these constructions knowing-wh: know followed by a wh-question word 3 The following table shows the number of hits returned by googling the corresponding terms 4 From the statistics, at least "know what"

Table 1 Hits (in millions) returned by google

\begin{tabular}{ccccccc}
\hline X & that & whether & what & how & who & why \\
\hline "know X" & 574 & 28 & 592 & 490 & 112 & 113 \\
"knows X" & 50.7 & 0.51 & 61.4 & 86.3 & 8.48 & 3.55 \\
\hline
\end{tabular}

and "know how" are equally frequent, if not more, as "know that" in natural language, and other expressions also play important roles in various contexts. Are those knowing-wh constructions as theoretically interesting as "knowing that"? Below we will briefly look at it from three different perspectives of linguistics, philosophy, and AI.

Linguists try to understand such constructions from a more general perspective in terms of classifications of verbs: which verbs can take an embedded wh-question? For example, forget, see, remember are like know in this sense. However, it is a striking cross-linguistic fact that the verb believe cannot take any of those embedded questions, in contrast with philosophers' usual conception of knowledge in terms of strengthened justified true belief. Linguists have been trying to give explanations in terms of factivity and other properties of verbs with interesting exceptions (cf. e.g., (Egré, 2008) and references therein). Moreover, when know is immediately followed by a noun phrase, it can usually be translated back to the knowing-wh constructions by treating the noun phrase as a concealed question, e.g., knowing the price of milk can be treated as knowing what the price of milk is (Heim, 1979). The semantic variability of the same knowing-wh-construction in different contexts also interest linguists a lot, e.g., "I know which card is the winning card" can mean I know Ace is the winning card for the game, or I know the card that my opponent holds is the winning card. There are approaches that give uniform treatments to handle this kind of context-sensitivity (cf. e.g., Aloni (2001)).

For philosophers, especially epistemologists, it is crucial to ask whether those knowing-wh statements are also talking about different kinds of knowledge. For

\footnotetext{
${ }^{3}$ How is in general also considered as a wh-question word, besides what, when, where, who, whom, which, whose, and why.

${ }^{4}$ The "knows X" search term can exclude the phrases such as "you know what" and count only the statements, while "know X" may appear in questions as well.
} 
example, it has been a frequently debated topic whether knowledge-how can be reduced to knowledge-that (cf. e.g., (Ryle, 1949; Stanley, 2011)). As another example, for philosophers of science, knowing why is extremely important, as it drives science forward. However, what amounts to knowing why? Many philosophers think knowing an scientific explanation is the key to answering why-questions, and there is a large body of research on it (cf. e.g., van Fraassen, 1980)). Knowing who also draws some attention from philosophers in analyzing the more general propositional attitude ascriptions, see (Boer and Lycan, 2003).

Already in the early days of AI, researchers realized knowing-wh statements are useful in specifying the precondition or the effects of actions (Moore, 1977). For example, it is crucial for a robot to know where to check or whom to ask, if it does not know what the email address of the person it wants to contact. McCarthy (1979) even considered knowing what as the most important type of knowledge in AI. Such knowing-wh statements also show up in various implemented AI systems, e.g., knowledge-based planning system (Petrick and Bacchus, 2004a b). Besides constructing knowledge bases, it is very handy to specify the goal of a system using knowing-wh constructions, e.g., knowing whether is used quite frequently to specify knowledge goals and precondition for actions.

So, what about epistemic logicians? In fact, Hintikka (1962) devoted the last chapter to "knowing who" in the context of quantified epistemic logic, for the reason that the agent names are already in the epistemic language that he introduced earlier. Hintikka believed other knowing-wh constructions can be treated alike with different sorts of constants in place 5 In fact he proposed to treat knowing-wh as "one of the first problems" in epistemic logic (Hintikka, 1989b). The formalism involves quantifiers that quantify into the modal scope which may cause ambiguity according to Quine 6 Hintikka had lengthy discussions on conceptual and technical problems of quantified epistemic logic and in fact gradually developed a more general epistemic logic which he called a "second generation epistemic logic" (Hintikka, 2003). However, the quantified epistemic logic did not draw as much attention as its propositional brother. As a result, the classic textbook by Fagin et al (1995) has only a very brief discussion of first-order epistemic logic, and in the handbook of epistemic logic by van Ditmarsch et al (2015), there is not much about quantifiers either. The only dedicated survey that we found for quantified epistemic logic is a section in a long paper on epistemic logic by Gochet and Gribomont (2006). It seems that the mainstream epistemic logicians mainly focus on the propositional cases. However, not only Hintikka himself did quite a lot of work on it but also there are fascinating new technical developments in quantified epistemic logic. This motivates the first part this paper: to give a brief overview on what Hintikka and others did about epistemic logics of knowing-wh and quantified epistemic logic in general.

\footnotetext{
5 Later on he singled out "knowing why" in his framework of interrogative models (Hintikka and Halonen, 1995).

${ }^{6}$ See (Garson, 2001) for a survey on the (technical) difficulties about quantification in modal logic.
} 
On the other hand, introducing quantifiers explicitly in the epistemic language has a high computational cost: many interesting quantified epistemic logics are not decidable. However, there is a way to go around this. In this paper we would like to propose a general quantifier-free approach to the logics of knowing-wh, which may balance expressivity and complexity. The central idea is simple: treat knowingwh construction as new modalities, just like Hintikka did for knowing that. This approach can avoid some of the technical and conceptual problems of the quantified epistemic logic due to its weak language. New techniques and logics are being developed as will be surveyed in the later part of the paper.

The rest of the paper is organized as follows: In Section 2.1 we survey Hintikka's various works on knowing-wh. Section 2.2 reviews the recent technical developments of quantified epistemic logic. Section 3 explains our new approach and its considerations. Section 4 gives three concrete examples to demonstrate our approach. In the last section we conclude with some further directions.

\section{Background in quantified epistemic logic}

\subsection{Hintikka on knowing-wh}

According to Hintikka (1989b), one of the most important applications of epistemic logic is to understand questions 7 A question "Who is $b$ ?" amounts to the request of information: bring about that I know who $b$ is. Hintikka called "I know who $b$ is" the desideratum of the corresponding question. Under this view, the study of questions reduces largely to the study of their corresponding desiderata. This interest in the relationship between questions and knowledge also led Hintikka to the pursuit of a Socratic epistemology that weighs knowledge acquisition more importantly than knowledge justification which has been the focus of the traditional epistemology (Hintikka, 2007).

To formalize "I know who $b$ is" we do need quantifiers. Hintikka (1962) proposed the formula $\exists x K(b=x)$, and compared it with $K \exists x(b=x)$ in order to demonstrate the distinction between de re and de dicto in the epistemic setting 8 He called the earlier one knowledge of objects and the later one propositional knowledge. However, once the constants and quantifiers are introduced into the language, we need a much richer structure over possible worlds. The possible worlds may not share the same domain of objects, for you may imagine something non-existent to exist

\footnotetext{
${ }^{7}$ It also makes sense to understand knowing-wh constructions by first understanding the semantics of questions, see (Harrah, 2002) and references therein. Knowing-wh is then knowing a/the answer of the corresponding wh-question.

${ }^{8}$ Hintikka (1962) argued that the quantification into the modal context is necessary and not misleading, in contrast to Quine who was against such quantification due to the lack of substitution of identity in modal context.
} 
in some possible world9 Now how do we "pick up" an object in order to evaluate the formula " $\exists x K(b=x)$ "? Hintikka (1989b) proposed to draw world-lines in different ways to identify objects across the world. His most important point here is that depending on how you draw the world-lines, the formulas like $\exists x K(b=x)$ may have different meanings. For example, $\exists x K(b=x)$ can mean I can visually identify a person, e.g., in a party scenario I can say I know who Bill is by pointing at someone: "just that guy over there!". According to Hintikka (1989b), this requires to draw perspectival world-lines to connect the visual images, which can sometimes be used to interpreted knowing who as acquaintance. On the other hand, we can draw public world-lines, which contribute to the semantics of knowing who by description, e.g., I know who Bill is: he is the mayor of this city and a well-know logician. We can also think that there are two kinds of quantifiers corresponding to these two ways of drawing the world-lines (Hintikka and Symons, 2003). Since the formalism of knowing-wh is still based on the knowing that operator $K_{i}$, Hintikka did not consider them as a different type of knowing (Hintikka, 2003).

Besides the simple knowing-wh sentences, there are natural knowing-wh expressions which involve predicates, e.g., "I know who murdered $b$ " can be formalized as $\exists x K M(x, b)$, which is the desideratum of the question "Who murdered $b$ ?". To fulfill the desideratum $\exists x K M(x, b)$, is it enough to have $K M(a, b)$ for some $a$ ? Hintikka (1989a) argued that merely knowing that $M(a, b)$ does not always lead to knowing who: the questioner should also know who $a$ actually is, which is called the conclusiveness condition. Indeed, answering the question "Who gave the first speech?" by "The first speaker." may not be informative at all. Of course it is debatable whether this requirement is pervasive in most of the contexts. From this point of view, the existential generalization rule may not hold: $K M(a, b)$ does not entail $\exists x K M(x, b)$.

It becomes more interesting when complicated knowing-wh sentences are considered. An example given by Hintikka (2003) is "I know whom every young mother should trust" (with the intention pointing to "her own mother"). It seems that we need to pick up the trusted one in a uniform way for each young mother, and thus $\exists f K \forall x(M(x) \rightarrow T(x, f(x)))$ is a faithful formalization. Actually such knowledge of functions is pervasive in empirical sciences, where the research can be viewed as asking Nature what is the (functional) dependence between different variables (Hintikka, 1999). For example, let $x$ be the controlled variable and $y$ be the observed variable, and we ask Nature the dependence between $x$ and $y$ by doing experiments $E$ by changing the value of $x$. The desideratum of such a question is that I know the dependence between $x$ and $y$ according to the experiments, which can be formalized as $\exists f K \forall x E(x, f(x))$ where $E$ can be viewed as the relation paring the values of $x$ and $y$ according to the experiments. Like before, merely having $K \forall x E(x, g(x))$ is not enough, we do need a conclusiveness condition that you know the function $g$ : $\exists f K \forall x(f(x)=g(x))$. In this way, Hintikka (1996) managed to explain how mathematical knowledge, such as the knowledge of certain functions, plays a role in empirical research.

9 How the domain varies may affect the corresponding quantified modal axioms, see (Braüner and Ghilardi, 2007) for a overview on this issue in first-order modal logic. 
However, the above discussion leads to the introduction of higher-order entities, whose existence is unclear (Hintikka, 2003). To avoid this problem, Hintikka made use of the idea prominent in the Independence Friendly Logic proposed by Hintikka and Sandu (1989). The idea is to introduce the independence sign "/" into the logic language to let some quantifiers jump out of the scopes of earlier ones, in order to have a branching structure of quantifiers which are linearly ordered in the formulas. For example, $\forall x(\exists y / \forall x)(x=y)$ is not valid anymore, compared to $\forall x \exists y(x=y)$, since the choice of $y$ is independent from the choice of $x$. Now the earlier "young mother" formula becomes $K \forall x(\exists y / K)(M(x) \rightarrow T(x, y))$ without the second-order quantification. Likewise, the desideratum of an experiment can be formalized as $K(\forall x)(\exists y / K) E(x, y)$. The slash sign not only works with quantifiers but also logical connectives. For example, $K(p(\vee / K) \neg p)$ expresses knowing whether $p$ while $K(p \vee \neg p)$ amounts to knowing a tautology. There is also a beautiful correspondence between the desideratum and the presupposition of the same whquestion. The desideratum can usually be obtained by adding a suitable slash in the corresponding presupposition. For example, the presupposition of "Who murdered $b$ ?" is that $K \exists x M(x, b)$, i.e., I know someone murdered $b$, and the desideratum is $K(\exists x / K) M(x, b)$ which is equivalent to $\exists x K M(x, b)$, i.e., I know who murdered $b$. Hintikka (2003) called the epistemic logic using such an extended language the second generation epistemic logic, for it can go beyond the first-order epistemic logic, although the apparent quantifications are still first-order 10

\subsection{Recent technical advances of quantified epistemic logic}

The only comprehensive survey on quantified epistemic logic that we found is the section 5 of a paper by Gochet and Gribomont (2006), which covers most of the important works up to the beginning of this century 11 Here we supplement it with some of the recent advances, which are, however, by no means exhaustive.

Most of the recent developments in quantified epistemic logic are applicationdriven. To handle cryptographic reasoning, Cohen and Dam (2007) propose a complete first-order epistemic logic with a counterpart semantics in order to model the indistinguishability of messages modulo one's decoding ability. To formalize the reasoning in games, Kaneko and Nagashima (1996) propose a first-order epistemic logic with common knowledge. Wolter (2000) shows that even very simple fragments of such a FO epistemic logic are not decidable. On the other hand, decidable fragments are found using techniques by Sturm et al (2000) based on the idea of monodic fragments of quantified modal logic, where only one free variable is al-

\footnotetext{
10 The above $K \forall x(\exists y / K)(M(x) \rightarrow T(x, y))$ is an example that cannot be expressed in standard first-order epistemic logic.

${ }^{11}$ For the background of first-order modal logic, the readers are referred to the handbook chapter by Braïner and Ghilardi (2007) and the book by Fitting and Mendelsohn (1998). For the discussions on the philosophical issues of quantified first-order epistemic logic, see Hollidav and Perry (2014) and references therein.
} 
lowed to appear in the scope of modalities. In a similar way, some monodic fragments of first-order temporal logic are proved decidable (cf. e.g., (Hodkinson et al, 2000, 2002; Hodkinson, 2002)). It also inspired Belardinelli and Lomuscio (2011) to discover useful fragments of FO epistemic temporal logic. FO epistemic temporal logic has also been used to verify security properties as demonstrated by Belardinelli and Lomuscio (2009, 2012).

In propositional epistemic logic, agent names are like rigid designators and they actually are indexes of the epistemic alternative relations in the model. However, this limits epistemic logic to a fixed, finite set of agents. Moreover, agents cannot have uncertainty about each other's identity. A natural extension is to allow (implicit) quantification over agents (Corsi, 2002; Corsi and Orlandelli, 2013; Corsi and Tassi, 2014), where different readings of a quantified modal formula can also be disambiguated. Another quantifying-over-agent approach appears in the context of rough sets with multiple sources (as agents) by Khan and Banerjee (2010).

It is also interesting to quantify over propositions, which leads to second-order epistemic logic by Belardinelli and van der Hoek (2015, 2016), built on an early work by Fine (1970). In such a framework, one can express that currently $i$ knows everything that $j$ knows 12 which was handled earlier in a different approach by van Ditmarsch et al (2012b).

Recent years also witness the growth of inquisitive semantics as an interdisciplinary field between linguistics and logic. It gives a uniform semantics to both descriptive and interrogative sentences (cf. e.g., (Ciardelli et al, 2013)). In such a framework, one can combine knowing that operator with an embedded interrogative compositionally, and this is how knowing whether is treated in the epistemic inquisitive logic (Ciardelli, 2014; Ciardelli and Roelofsen, 2015). The readers are referred to the $\mathrm{PhD}$ thesis by Ciardelli (2016) for recent developments.

\section{Epistemic logics of knowing-wh: a new proposal}

Our point of departure from the aforementioned existing research is that we take a knowing-wh construction as a single modality, just like $K$ for knowing that, without explicitly introducing quantifiers, predicates, and equality symbols into the logic language. For example, instead of rendering "agent $i$ knows what the value of $c$ is" as $\exists x K_{i}(c=x)$, we simply have $K v_{i} c$ where $K v_{i}$ is a new knowing what modality. An example language of knowing what is as follows (to be discussed in detail later):

$$
\varphi::=\top|p| \neg \varphi|(\varphi \wedge \varphi)| K_{i} \varphi \mid K v_{i} c
$$

where $c$ belongs to a set $\mathbf{C}$ of constant symbols.

Following Hintikka, we take a semantics-driven approach for there is usually not enough syntactic intuition on the possible axioms for such knowing-wh con-

${ }^{12}$ Modeling it globally can be done in propositional modal logic with new axioms like $K_{j} p \rightarrow K_{i} p$, cf. e.g., Lomuscio and Ryan (1999). 
structions. We can discover interesting axioms by axiomatizing the valid formuals w.r.t. the semantics. The models are usually richer than Kripke models for propositional epistemic logic. For example, the semantics for $K v_{i} c$ is given over firstorder Kripke models with a constant domain: $\mathscr{M}=\left\langle S, D,\left\{\rightarrow_{i} \mid i \in \mathbf{I}\right\}, V, V_{C}\right\rangle$ where $\left\langle S,\left\{\rightarrow_{i} \mid i \in \mathbf{I}\right\}, V\right\rangle$ is a usual Kripke model, $D$ is a constant domain of values (all the worlds share the same $D$ ), and $V_{\mathbf{C}}: \mathbf{C} \times S \rightarrow D$ assigns to each (non-rigid designator) $c \in \mathbf{C}$ a $d \in D$ on each $s \in S$ :

$\mathscr{M}, s \vDash K v_{i} c \Leftrightarrow$ for any $t_{1}, t_{2}:$ if $s \rightarrow_{i} t_{1}, s \rightarrow_{i} t_{2}$, then $V_{C}\left(c, t_{1}\right)=V_{C}\left(c, t_{2}\right)$.

Intuitively, $i$ knows what the value of $c$ is iff $c$ has the same value over all the $i$ accessible worlds. This is the same as the semantics for $\exists x K_{i}(c=x)$ on constant domain FO Kripke models. We will come back to the details in Section 4.2

After defining the language and semantics, we can try to find a complete axiomatization with meaningful axioms, and then dynamify the logic to include updates of knowledge as in dynamic epistemic logic (van Ditmarsch et al, 2007). The axioms will tell us some intrinsic logical features of the knowing-wh construction. We may come back to philosophy with new insights after finishing the formal work.

Such an approach has the following advantages:

Neat language and characterizing axioms Using knowing-wh modalities can make the formal languages very simple yet natural, which can also highlight the logical differences between different knowing-wh in terms of intuitive axioms, e.g., knowing whether $\varphi$ is equivalent to knowing whether $\neg \varphi$. It will also become clear how knowing-wh modalities differ from the normal modalities, e.g., knowing how to achieve $\varphi$ and knowing how to achieve $\psi$ does not entail knowing how to achieve $\varphi \wedge \psi$ (e.g., take $\psi=\neg \varphi$ ).

Balancing expressivity and complexity The new languages may be considered as small fragments of quantified epistemic logic and we can try to balance the expressivity and complexity. For example, the above $K v$ modality packages a quantifier, a $K$ modality, and an equality together. Such a packed treatment is also the secret of the success of standard modal logic, where a quantifier and a relational guard are packed in a modality. Such weaker languages are in general more applicable in practice due to computational advantages. Our approach may also help to discover new decidable fragments of quantified modal logics.

Avoiding some conceptual problems The history of epistemic logic taught us a lesson that the logical framework can be extremely useful even before philosophers reach a consensus on all its issues, if they ever do so at all. Certain conceptual difficulties about quantified epistemic logic should not stop us from developing the logic further while bearing those questions in mind, since new insights may come as you start to move forward. Our weaker languages are free of explicit quantifiers, thus it may avoid some difficulties in the full quantified epistemic logic and makes us focus on the limited but reasonably clear fragments 13

${ }^{13}$ The absence of equality symbols also make the substitution of equal constants apparently irrelevant. 
Connections to existing modal logics As we will see, each of the knowing-wh logics has some very close (sometimes surprising) friends in propositional modal logic. We may benefit from the vast existing results and tool support for propositional modal logic. As we will see, the new operators can also motivate new ways to update the models which were not considered before.

Of course, there are also limitations and difficulties of this approach:

- The languages cannot express knowing-wh constructions in a fully compositional way when complicated constructions are involved, e.g., John knows what Mary knows about logic. Also from the linguistic perspective, our approach cannot handle context-sensitivity of the meaning of the knowing-wh constructions 14

- Our languages are relatively weak, but the models are very rich in order to accommodate an intuitive semantics. This apparent asymmetry between syntax and semantics may cause difficulties in axiomatizating the logics. However, we may restore the symmetry by simplifying the models modulo the same logic once we have a complete axiomatization w.r.t. the rich models.

- From a syntactic point of view, the new logics are usually not normal, e.g., knowing whether $\varphi \rightarrow \psi$ and knowing whether $\varphi$ does not entail knowing whether $\psi$, for you may know that $\varphi$ is false but have no idea about the truth value of $\psi 15$

- Although many knowing-wh modalities share a general form of $\exists x K \varphi(x)$, different modalities can still behave quite differently depending on the exact shape of $\varphi(x)$. Also, the existential quantifier may not necessarily be first-order as in the later example of a logic of knowing how.

- In some cases it is highly non-trivial to give a reasonable semantics since we do not understand enough about the meaning of certain knowing-wh yet.

In the following we give three example studies on knowing-wh to demonstrate the claimed advantages, and how we overcome some of the technical difficulties mentioned above.

\section{Examples}

In this section, we demonstrate the use and techniques of the proposed approach with three examples: the logics of knowing whether, knowing what, and knowing how. Besides the historical background and the common pursuit for complete axiomatizations, each example has its own special focus to give the readers a more general picture of the approach. The readers may pay attention to the points below.

- Knowing whether: expressivity comparisons over models and frames w.r.t. standard modal logic, and completeness proof for such non-normal modal logic;

\footnotetext{
${ }^{14}$ See (Aloni, 2016) in this volume for a quantified epistemic logic treatment of this contextsensitivity of knowing who, using conceptual covers proposed by Aloni (2001).

15 On the other hand, a slightly different axiom holds intuitively: knowing whether $\varphi \leftrightarrow \psi$ and knowing whether $\varphi$ does entail knowing whether $\psi$.
} 
- Knowing what: interaction axioms between knowing that and the new modality, conditionalization of the new modality, asymmetry between syntax and semantics and the techniques to restore the symmetry, and a new update operation;

- Knowing how: philosophically inspired language design, AI inspired semantics design, epistemic models without epistemic relations, and techniques of completeness proof when $x$ is not unique (nor first-order) to make $\exists x K \varphi(x)$ true.

Impatient readers who only want to see one example may jump to Section 4.2 on a logic of knowing what since it is the most representative one for the proposed approach. In the following examples, we will focus on the ideas behind definitions and results rather than technical details, which can be found in the cited papers.

\subsection{Knowing whether}

The logic of knowing-whether is perhaps the closest knowing-wh friend of the standard epistemic logic, yet it can already demonstrate many shared features of the logics of knowing-wh. Although it is clear that knowing whether $\varphi\left(K w_{i} \varphi\right)$ is equivalent to knowing that $\varphi$ or knowing that $\neg \varphi$, introducing the knowing whether operator firstly has an advantage in succinctness, as van Ditmarsch et al (2014) showed. In many epistemic puzzles such as muddy children, the goal and the preconditions of actions are often formulated as knowing whether formulas. As a philosophical example, van Ditmarsch et al (2012a) showed that although it is not possible to know every true proposition according to Fitch's paradox based on Moore sentences 16 everything is eventually knowable in terms of knowing whether it is true (the truth value may have changed). It also makes sense to iterate the knowing whether operators of different agents to succinctly capture the higher-order observability of agents towards each other, e.g., I know whether you know whether $p$ although I do not know whether $p$ (cf. e.g., the sees operator by Herzig et al (2015)). As a technical example, Hart et al (1996) made use of the alternations of knowing whether operators to neatly build $2^{\aleph_{0}}$ many mutually inconsistent knowledge states of two agents, which greatly simplified a previous construction by Aumann (1989) using knowing that operators. The construction of Hart et al (1996) relies on an intuitive axiom about knowing whether: $K w_{i} \varphi \leftrightarrow K w_{i} \neg \varphi$. Now, what is the complete axiomatic system for the logic of knowing whether, where $K w_{i}$ is the only primitive modality? How is the expressivity of this logic compared to that of the standard epistemic /modal logic?

Actually, such technical questions have been partly addressed under the name of non-contingency logic where the modality symbol $\Delta$ takes the place of $K w$, which we will follow from now on. Indeed, if you view the modal operator $\square$ as a necessity operator then $\Delta \varphi:=\square \varphi \vee \square \neg \varphi$ says that $\varphi$ is not contingent. In differ-

\footnotetext{
${ }^{16}$ Fitch proved that you cannot know all the truths, e.g., $p \wedge \neg K_{i} p$ is not knowable by $i$, which is demonstrated by the inconsistent Moore sentence: $K_{i}\left(p \wedge \neg K_{i} p\right)$ in the basic epistemic logic, see (Fitch, 1963).
} 
ent contexts this operator has different readings. In the context of alethic modality, the study of contingency logic goes back to Montgomery and Routley (1966) and involves the works of many well-known logicians 17 in the epistemic context, it amounts to knowing whether 18 and its negation amounts to a notion of ignorance (van der Hoek and Lomuscio, 2004); in the doxastic setting, $\Delta \varphi$ says that the agent is opinionated about $\varphi$; in the deontic setting, $\neg \Delta \varphi$ means moral indifference (Von Wright, 1951); in the proof theoretical context, $\neg \Delta \varphi$ means that $\varphi$ is undecided (Zolin, 2001). In different settings, different frame conditions may be imposed, thus it is interesting to see how the logic behaves over different frame classes, as in standard modal logic. In the following, let us get a taste of this simple yet interesting language by looking into a few formal results.

\subsubsection{Language, semantics and expressivity}

Following the tradition in non-contingency logic, call the following language NCL:

$$
\varphi::=\top|p| \neg \varphi|(\varphi \wedge \varphi)| \Delta_{i} \varphi
$$

where $p \in \mathbf{P}$ and $i \in \mathbf{I}$. It is interpreted on Kripke models $\mathscr{M}=\left\langle S,\left\{\rightarrow_{i} \mid i \in \mathbf{I}\right\}, V\right\rangle, 19$

$$
\begin{aligned}
\mathscr{M}, s \vDash \Delta_{i} \varphi & \Leftrightarrow \text { for all } t_{1}, t_{2} \text { such that } s \rightarrow_{i} t_{1}, s \rightarrow_{i} t_{2}:\left(\mathscr{M}, t_{1} \vDash \varphi \Leftrightarrow \mathscr{M}_{,} t_{2} \vDash \varphi\right) \\
& \Leftrightarrow \text { either for all } t \text { s.t. } s \rightarrow_{i} t: \mathscr{M}, t \vDash \varphi \text { or for all } t \text { s.t. } s \rightarrow_{i} t: \mathscr{M}, t \not \models \varphi
\end{aligned}
$$

Note that we do not impose any properties on the frames unless specified. NCL is clearly no more expressive than the standard modal logic (ML) since we can define a translation $t: \mathbf{N C L} \rightarrow \mathbf{M L}$ such that: $t\left(\Delta_{i} \varphi\right)=\square_{i} t(\varphi) \vee \square_{i} \neg t(\varphi)$. What about the other way around? If we restrict ourselves to reflexive models, we can also define a translation $t^{\prime}: \mathbf{M L} \rightarrow \mathbf{N C L}$, namely $t^{\prime}\left(\square_{i} \varphi\right)=t^{\prime}(\varphi) \wedge \Delta_{i} t^{\prime}(\varphi)$. However, NCL and ML do not have the same expressive power over arbitrary models. We can use a notion of bisimulation to measure the expressive power of the logic. Let us first recall the standard definition of bisimulation in modal logic:

Definition 1 (Bisimulation). Let $\mathscr{M}=\left\langle S,\left\{\rightarrow_{i} \mid i \in \mathbf{I}\right\}, V\right\rangle, \mathscr{N}=\left\langle S^{\prime},\left\{\rightarrow_{i}^{\prime} \mid i \in \mathbf{I}\right\}, V^{\prime}\right\rangle$ be two models. A binary relation $Z$ over $S \times S^{\prime}$ is a bisimulation between $\mathscr{M}$ and $\mathscr{N}$, if $Z$ is non-empty and whenever $s Z s^{\prime}$ :

- (Invariance) $s$ and $s^{\prime}$ satisfy the same propositional variables;

- (Zig) if $s \rightarrow_{i} t$, then there is a $t^{\prime}$ such that $s^{\prime} \rightarrow_{i} t^{\prime}$ and $t Z t^{\prime}$;

- (Zag) if $s^{\prime} \rightarrow_{i} t^{\prime}$, then there is a $t$ such that $s \rightarrow_{i} t$ and $t Z t^{\prime}$.

$\mathscr{M}, s$ is bisimilar to $\mathscr{N}, t(\mathscr{M}, s \overleftrightarrow{N}, t)$ if there is a bisimulation between $\mathscr{M}$ and $\mathscr{N}$ linking $s$ with $t$.

${ }^{17}$ For example, Cresswell (1988); Kuhn (1995); Humberstone (1995); Demri (1997); Zolin (1999); Pizzi (2007), see (Fan et al, 2015) for a survey.

${ }^{18}$ See Aloni et al 2013) for more general versions of the knowing whether operator.

${ }^{19}$ Similar semantics has been applied to neighborhood structures (Fan and van Ditmarsch, 2015). 
It is well-known that modal logic is invariant under bisimilarity, thus bisimilarity is also an invariance relation for NCL. However, it is too strong even on finite models. The two pointed models $\mathscr{M}, s$ and $\mathscr{N}, s^{\prime}$ below satisfy the same NCL formulas but they are not bisimilar20

$$
\underline{s}: p \longrightarrow i \rightarrow t: p \quad \underline{s^{\prime}}: p
$$

However, in most of the cases when there are two or more successors standard bisimilarity works fine. To tell the subtle difference we need to connect $\Delta$ with $\square$. Fan et al (2014) have a crucial observation that $\square_{i}$ is almost definable by $\Delta_{i}$.

Proposition 1 (Almost-definability Schema (AD) Fan et al (2014)). For any $\varphi, \psi$ in the modal language with both $\square_{i}$ and $\Delta_{i}$ modalities:

$$
\vDash \neg \Delta_{i} \psi \rightarrow\left(\square_{i} \varphi \leftrightarrow\left(\Delta_{i} \varphi \wedge \Delta_{i}(\psi \rightarrow \varphi)\right)\right) .
$$

The idea is that if there are two $i$-accessible worlds differentiated by a formula $\psi$, then $\square_{i}$ is locally definable in terms of $\Delta_{i}$. The missing part between $\square_{i} \varphi$ and $\Delta_{i} \varphi$ is that we need to force $\varphi$, instead of $\neg \varphi$, to hold over the $i$-accessible worlds, and the contingency of $\psi$ helps to fill in the gap. This almost-definability schema (AD) inspires us to find:

- a notion of $\Delta_{i}$-bisimulation which characterizes the expressive power of NCL;

- the suitable definition of canonical relations in the completeness proofs;

- the right axioms for special frame properties.

From AD, if there are two states which can be told apart by a NCL formula then the standard bisimulation should work locally. However, to turn this precondition into a purely structural requirement is quite non-trivial. The idea is to define the bisimulation notion within a single model and then generalize the bisimilarity notion using disjoint unions of two models.

Definition 2 ( $\Delta$-Bisimulation). Let $\mathscr{M}=\left\langle S,\left\{\rightarrow_{i} \mid i \in \mathbf{I}\right\}, V\right\rangle$ be a model. A binary relation $Z$ over $S$ is a $\Delta$-bisimulation on $\mathscr{M}$, if $Z$ is non-empty and whenever $s Z s^{\prime}$ :

- (Invariance) $s$ and $s^{\prime}$ satisfy the same propositional variables;

- (Zig) if there are two different successors $t_{1}, t_{2}$ of s such that $\left(t_{1}, t_{2}\right) \notin Z$ and $s \rightarrow_{i} t$, then there exists $t^{\prime}$ such that $s^{\prime} \rightarrow_{i} t^{\prime}$ and $t Z t^{\prime}$;

- (Zag) if there are two different successors $t_{1}^{\prime}, t_{2}^{\prime}$ of $s^{\prime}$ such that $\left(t_{1}^{\prime}, t_{2}^{\prime}\right) \notin Z$ and $s^{\prime} \rightarrow_{i} t^{\prime}$, then there exists $t$ such that $s \rightarrow_{i} t$ and $t Z t^{\prime}$.

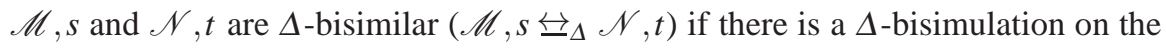
disjoint union of $\mathscr{M}$ and $\mathscr{N}$ linking $s$ and $t$.

In contrast to the standard bisimilarity, to show that $\Delta$-bisimilarity is indeed an equivalence relation is not at all trivial but a good exercise to appreciate better the definition 21

\footnotetext{
${ }^{20}$ Note that if there is at most one successor of $s$ then every $\Delta \varphi$ formula holds.

21 The transitivity is hard, you need to enrich the two bisimulations a bit in connection with the middle model when proving it, see (Fan, 2015).
} 
Based on $\Delta$-bisimilarity, Fan et al (2014) proved:

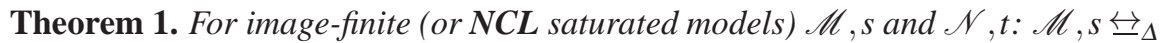
$\mathscr{N}, t \Longleftrightarrow \mathscr{M}, s \equiv_{N C L} \mathscr{N}, t$ (satisfying the same $N C L$ formulas).

Theorem 2. NCL is the $\triangle$-bisimilarity invariant fragment of $\mathrm{ML}$ (and $\mathrm{FOL}$ ).

The proof mimics the standard proofs in modal logic by using AD repeatedly to simulate $\square$ whenever possible.

A natural question arises: if you can almost always define $\square$ using $\Delta$ locally on models, is the difference in expressivity just a negligible subtlety? However, Fan et al (2014) showed that in terms of frame definability it is a significant difference.

Theorem 3. The frame properties of seriality, reflexivity, transitivity, symmetry, and Euclidicity are not definable in NCL.

The proof by Fan et al (2014) uses the following frames:

$$
\mathscr{F}_{1} \quad s_{1} \longrightarrow t \longrightarrow u \quad \mathscr{F}_{2} \quad s_{2}
$$

It can be shown that $\mathscr{F}_{1} \vDash \varphi \Longleftrightarrow \mathscr{F}_{2} \vDash \varphi$ for all NCL-formula $\varphi$, based on the invariance under $\Delta$-bisimilarity and possible valuations over the frames. However, the left frame is not reflexive (transitive, serial, symmetric and Euclidean) while the right one has all these properties. Therefore such frame properties are not definable. This presents a sharp difference between NCL and ML, and this may cause difficulties in axiomatizing NCL over different frame classes.

\subsubsection{Axiomatizations}

In axiomatizing NCL over different frame classes to apply it in different contexts, we apparently face the following difficulties:

- It is impossible to use NCL formulas to capture frame properties.

- NCL is not normal, e.g., $\Delta_{i}(\varphi \rightarrow \psi) \wedge \Delta_{i} \varphi \rightarrow \Delta_{i} \psi$ is invalid, as mentioned before.

- NCL is also not strictly weaker than modal logic, i.e. $\Delta_{i} \varphi \leftrightarrow \Delta_{i} \neg \varphi$ is valid.

The following system $\mathbb{S N C L}$ is proposed by Fan et al (2014, 2015)

\begin{tabular}{lll}
\multicolumn{1}{c}{ System $\mathbb{S N C L}$} & \\
Axioms & Rules \\
TAUT all the instances of tautologies & $\operatorname{MP}$ & $\frac{\varphi, \varphi \rightarrow \psi}{\psi}$ \\
KwCon $\Delta_{i}(q \rightarrow p) \wedge \Delta_{i}(\neg q \rightarrow p) \rightarrow \Delta_{i} p$ & $\mathrm{NEC}$ & $\frac{\varphi}{\Delta_{i} \varphi}$ \\
$\mathrm{KwDis} \Delta_{i} p \rightarrow \Delta_{i}(p \rightarrow q) \vee \Delta_{i}(\neg p \rightarrow q)$ & $\mathrm{SUB}$ & $\frac{\varphi}{\varphi[p / \psi]}$ \\
$\mathrm{Kw} \leftrightarrow$ & $\operatorname{REPL} \frac{\varphi \leftrightarrow \psi}{\Delta_{i} \varphi \leftrightarrow \Delta_{i} \psi}$
\end{tabular}

\footnotetext{
${ }^{22}$ See Fan et al (2015) for comparisons with other equivalent systems in the literature.
} 
KwCon tells us how to derive $\Delta_{i} \varphi$, and $\operatorname{KwDi}$ is tells us how to derive from $\Delta_{i} \varphi$. $\mathrm{KwCon}$ is actually useful if we take it as a guide for the questioning strategy aiming at knowing whether $p$ (cf. e.g., (Liu and Wang, 2013)). Imagine that a student $i$ wants to know whether he has passed the exam $(p)$ or not, but does not want to ask the teacher directly. According to the axiom, he can ask the teacher two apparently innocent questions related to whether someone else (say $j$ ) has passed the exam $(q)$ : (1) "Is it the case that $j$ or I passed the exam?" (to obtain $\Delta_{i}(q \vee p)$, i.e., $\Delta_{i}(\neg q \rightarrow p)$ ) and (2) "Is it the case that if $j$ passes then I pass too?" (to obtain $\Delta_{i}(q \rightarrow p)$ ). By axiom KwCon, $\Delta_{i} p$ then holds 23 Note that since the distribution axiom no longer holds for $\Delta_{i}$, we need the replacement rule REPL to facilitate the substitution of equivalent formulas.

Theorem 4 (Fan et al (2015)). SNCL is sound and strongly complete w.r.t. NCL over the class of arbitrary frames.

The completeness proof is based on the following canonical model construction, inspired by the almost-definability schema again.

Definition 3 (Canonical model). Define $\mathscr{M}^{c}=\left\langle S^{c}, R^{c}, V^{c}\right\rangle$ as follows:

- $S^{c}=\{s \mid s$ is a maximal consistent set of $\mathbb{S N C L}\}$

- For all $s, t \in S^{c}, s R_{i}^{c} t$ iff there exists $\chi$ such that:

- $\neg \Delta_{i} \chi \in s$, and

- for all $\varphi, \Delta_{i} \varphi \wedge \Delta_{i}(\chi \rightarrow \varphi) \in s$ implies $\varphi \in t$.

- $V^{c}(p)=\left\{s \in S^{c} \mid p \in s\right\}$.

Readers who are familiar with modal logic can immediately see the similarity to the standard definition of canonical relations: $\Delta_{i} \varphi \wedge \Delta_{i}(\chi \rightarrow \varphi)$ acts as $\square_{i} \varphi$ given $\neg \Delta_{i} \chi \in s$. Note that if $\Delta_{i} \chi \in s$ for every NCL-formula $\chi$ then there is simply no need to have an outgoing arrow from $s$.

In the proof of the truth lemma, the hard part is to show that $\Delta_{i} \psi \notin s$ implies $\mathscr{M}^{c}, s \not \models \Delta_{i} \psi$. Here it is worthwhile to stress a characteristic feature which is shared by some other knowing-wh logics. Note that to show $\mathscr{M}^{c}, s \not \models \Delta_{i} \psi$ (existence lemma), we need to construct two successors of $s$ such that $\psi$ holds on one and does not hold on the other. Bearing the schema AD in mind, it boils down to show the following two sets are consistent, which can be proved using the axioms:

1. $\left\{\varphi \mid \Delta_{i} \varphi \wedge \Delta_{i}(\psi \rightarrow \varphi) \in s\right\} \cup\{\psi\}$ is consistent.

2. $\left\{\varphi \mid \Delta_{i} \varphi \wedge \Delta_{i}(\neg \psi \rightarrow \varphi) \in s\right\} \cup\{\neg \psi\}$ is consistent.

For NCL over other frame classes, Fan et al (2015) present all the complete axiomatizations based on $\mathbb{S N C L}$ in Table 24 Note that although wKw4 and wKw5 look like the corresponding axioms 4 and 5 of standard epistemic logic, $\mathbb{S N C L}+w K w 4$ and $\mathbb{S N C L}+$ wKw5 are not complete over the classes of transitive and euclidean

${ }^{23}$ Here we can also see the parallel of deduction and interrogation that Hintikka (2007) discussed.

${ }^{24}$ For some equivalent proof systems in the literature, see the survey and comparisons in Fan et al, 2015). 
Table 2 Axiomatizations of NCL over various frame classes

\begin{tabular}{|c|c|c|c|}
\hline Notation & Axiom Schemas & Systems & Frames \\
\hline KWT & $\overline{\Delta_{i} \varphi \wedge \Delta_{i}(\varphi \rightarrow \psi) \wedge \varphi \rightarrow \Delta_{i} \psi}$ & $\mathbb{S N C L T}=\mathbb{S N C L}+K w T$ & reflexive \\
\hline Kw4 & $\Delta_{i} \varphi \rightarrow \Delta_{i}\left(\Delta_{i} \varphi \vee \psi\right)$ & $\mathbb{S N C L} 4=\mathbb{S N C L}+\mathrm{Kw} 4$ & transitive \\
\hline Kw5 & $\neg \Delta_{i} \varphi \rightarrow \Delta_{i}\left(\neg \Delta_{i} \varphi \vee \psi\right)$ & $\mathbb{S N C L} 5=\mathbb{S N C L}+\mathrm{Kw} 5$ & euclidean \\
\hline wKw 4 & $\Delta_{i} \varphi \rightarrow \Delta_{i} \Delta_{i} \varphi$ & $\mathbb{S N C L S} 4=\mathbb{S N C L}+\mathrm{KwT}+\mathrm{wKw} 4$ & ref. \& trans. \\
\hline wKw5 & $\neg \Delta_{i} \varphi \rightarrow \Delta_{i} \neg \Delta_{i} \varphi$ & $\mathbb{S N C L S 5}=\mathbb{S N C L}+\mathrm{KwT}+\mathrm{wKw5}$ & equivalence \\
\hline KwB & $\begin{array}{l}\varphi \rightarrow \Delta_{i}\left(\left(\Delta_{i} \varphi \wedge \Delta_{i}(\varphi \rightarrow \psi)\right.\right. \\
\left.\left.\wedge \neg \Delta_{i} \psi\right) \rightarrow \chi\right)\end{array}$ & $\mathbb{S N C L B}=\mathbb{S N C L}+\mathrm{KwB}$ & symmetric \\
\hline
\end{tabular}

frames respectively. We need their stronger versions. On the other hand, in presence of KwT, wKw 4 and wKw5 are enough to capture NCL over $\mathbb{S} 5$ frames.

Here are two points we want to stress (details can be found in (Fan et al, 2015)):

- We may find new axioms by using the almost-definability schema to translate the standard modal logic axioms corresponding to the frame properties.

- The axioms are usually not canonical but we can transform the canonical model into the right shape.

We conclude our discussion on knowing whether by adding public announcements to NCL:

$$
\varphi::=\top|p| \neg \varphi|(\varphi \wedge \varphi)| \Delta_{i} \varphi \mid[\varphi] \varphi
$$

with the standard semantics as in public announcement logic of Plaza (1989):

$$
\mathscr{M}, s \vDash[\psi] \varphi \Leftrightarrow \mathscr{M}, s \vDash \psi \text { implies }\left.\mathscr{M}\right|_{\psi, s} \vDash \varphi
$$

where $\left.\mathscr{M}\right|_{\psi}=\left(S^{\prime},\left\{\rightarrow_{i}^{\prime} \mid i \in \mathbf{I}\right\}, V^{\prime}\right)$ such that: $S^{\prime}=\{s \mid \mathscr{M}, s \vDash \psi\}, \rightarrow_{i}^{\prime}=\left.\rightarrow_{i}\right|_{S^{\prime} \times S^{\prime}}$ and $V^{\prime}(p)=V(p) \cap S^{\prime}$.

With the usual reduction axioms and the following one, Fan et al (2015) axiomatized the extended logic over various classes of frames:

$$
[\varphi] \Delta_{i} \psi \leftrightarrow\left(\varphi \rightarrow\left(\Delta_{i}[\varphi] \psi \vee \Delta_{i}[\varphi] \neg \psi\right)\right)
$$

A similar story holds if we introduce the event model modality in DEL Fan et al, 2015). By having both the updates and knowing whether modalities in place, this simple language can be used to model the goal and the preconditions of actions in the scenarios of epistemic planning with polar questions/binary tests. For example, in a version of muddy children, the father asks "Please step forward, if you know whether you are dirty". After repeating the announcement several times, all the dirty children know whether they are dirty.

Instead of the standard announcement operator, we can also introduce the announcing whether operator $[? \varphi]$ which updates the model with the $\varphi$ or $\neg \varphi$ depending on the actual truth value of $\varphi$ (cf. e.g., Van Ditmarsch et al, 2011; van Ditmarsch and Fan, 2016)). It is easy to see that $[? \varphi] \psi \leftrightarrow([\varphi] \psi \wedge[\neg \varphi] \psi)$. This operator may be useful in presenting protocols involving telling the truth value of a proposition such as 
the protocol for dining cryptographers (Chaum, 1988). In the next section, we will generalize this idea to announcing the value of a constant.

\subsection{Knowing what}

Knowing whether $\varphi$ can also be viewed as knowing what the truth value of $\varphi$ is. In this subsection, we survey the line of work on a simple yet ubiquitous type of knowing what: "knowing [what the] value [is]" where each constant has a value that ranges over a possibly infinite domain 25 Note that since the domain may be infinite, it does not make sense to encode knowing the value of $c$ by the disjunction of knowing that $c=v_{1}$, knowing that $c=v_{2}$, and so on. This is a fundamental difference between knowing whether and knowing value, which makes the latter much more interesting.

The study of knowing value as a modal operator dates back to (Plaza, 1989), which is well-known for the invention of public announcement logic (PAL) 26 Interestingly enough, almost one half of this classic paper was devoted not to "knowing that" but to "knowing value", which was, to our knowledge, largely neglected by the later literature except the comments by van Ditmarsch (2007). Plaza (1989) used two running examples to demonstrate the update effects of public announcements: the muddy children and the sum-and-product puzzle 27 To model the second puzzle, Plaza (1989) introduced a special $K v_{i}$ modality to the epistemic language to express that agent $i$ knows the value of some constant. Let us call the following language $\operatorname{PALKv}$ (where $c$ is any constant symbol in a given set $\mathbf{C}$ ):

$$
\varphi::=\top|p| \neg \varphi|(\varphi \wedge \varphi)| K_{i} \varphi\left|K v_{i} c\right|[\varphi] \varphi
$$

We use the usual abbreviations $\hat{K}_{i}$ and $\langle\varphi\rangle$ for the diamond versions of $K_{i}$ and $[\varphi]$.

By having both $K_{i}$ and $K v_{i}$, PALKv can express interesting interactions between them, e.g., " $i$ knows that $j$ knows the password but $i$ doesn't know what exactly it is" by $K_{i} K v_{j} c \wedge \neg K v_{i} c$. Note that replacing $K v$ by $K$ and replacing constant $c$ by a proposition $p$ will result in an inconsistent formula $K_{i} K_{j} p \wedge \neg K_{i} p 28$

In contrast to the in-depth study of public announcement logic, Plaza did not give the axiomatization of the above logic with both announcement and the $K v$ operator but only a few axioms on top of $\mathbb{S} 5$, and this was the starting point of the study by

\footnotetext{
${ }^{25}$ As we mentioned earlier, knowing the value can be seen as knowing the answer to a concealed question, see Aloni and Roelofsen (2011) and references therein for some recent discussions.

${ }^{26}$ A similar definition of the knowing value modal operator appeared earlier in Ma and Guo (1983) as an abbreviation in a setting of quantified epistemic logic.

27 Two people S and P are told respectively the sum and product of two natural numbers which are known to be below 100. The following conversation happens: P says: "I do not know the numbers." S says: "I knew you didn’t." P says: "I now know the numbers.” S says: "I now also know it."

${ }^{28}$ On the other hand, replacing $K v$ with the knowing whether operator results in a consistent formula.
} 
Wang and Fan 2013). It turns out that those axioms are not enough to capture the logic w.r.t. the semantics we mentioned at the beginning of Section 4 for $K v_{i}$ :

Theorem 5 (Wang and Fan (2013)). The valid formula $\langle p\rangle K v_{i} c \wedge\langle q\rangle K v_{i} c \rightarrow\langle p \vee$ $q\rangle K v_{i} c$ is not derivable in the $\mathbb{S} 5$ system with Plaza's new axioms.

By defining a suitable bisimulation notion, Wang and Fan (2013) showed that PALKv is not reducible to its announcement-free fragment ELKv, thus the standard reductive-technique of dynamic epistemic logic cannot work here: you can never use reduction axioms to capture the logic of PALKv based on a system of the epistemic logic with $K v_{i}$ but not announcements 29 In the following, we propose an apparently more general conditional $K v_{i}$ operator that can encode the public announcements with reduction axioms. We believe the generalized operators constitute a language which is easier to use.

\subsubsection{Language, semantics and expressivity}

We start with a conditional generalization of $K v_{i}$ operator introduced by Wang and Fan (2013) (call the language $\mathbf{E L K \mathbf { V } ^ { r }}$ where ${ }^{r}$ means "relativized"):

$$
\varphi::=\top|p| \neg \varphi|(\varphi \wedge \varphi)| K_{i} \varphi \mid K v_{i}(\varphi, c)
$$

where $K v_{i}(\varphi, c)$ says "agent $i$ knows what $c$ is given $\varphi$ ". For example, I may forget my login password for a website, but I can still say that I know what the password is given that it is four-digit, since I have only one four-digit password ever. Actually, everyday knowledge is usually conditional 30 As mentioned earlier, the semantics is based on first-order epistemic models with a constant domain $\mathscr{M}=\left\langle S, D,\left\{\sim_{i} \mid i \in\right.\right.$ I $\left.\}, V, V_{C}\right\rangle$ where $\sim_{i}$ is an equivalence relation:

$$
\begin{aligned}
\mathscr{M}, s \vDash K v_{i}(\varphi, c) \Leftrightarrow & \text { for any } t_{1}, t_{2} \in S \text { such that } s \sim_{i} t_{1} \text { and } s \sim_{i} t_{2}: \\
& \mathscr{M}, t_{1} \vDash \varphi \text { and } \mathscr{M}, t_{2} \vDash \varphi \text { implies } V_{C}\left(c, t_{1}\right)=V_{C}\left(c, t_{2}\right)
\end{aligned}
$$

Intuitively, the semantics says that $i$ knows the value of $c$ given $\varphi$ iff on all the $\varphi$ worlds that he considers possible, $c$ has exactly the same value. The announcement operator can also be added to $\mathbf{E L K v}{ }^{r}$ and obtain PALKv ${ }^{r}$ :

$$
\varphi::=\top|p| \neg \varphi|(\varphi \wedge \varphi)| K_{i} \varphi\left|K v_{i}(\varphi, c)\right|[\varphi] \varphi
$$

PALKv ${ }^{r}$ looks more expressive than PALKv, but in fact both logics are equally expressive as the announcement-free $\mathbf{E L K} \mathbf{v}^{r}$ :

Theorem 6 (Wang and Fan (2013)). The comparison of the expressive power of those logics are summarized in the following (transitive) diagram:

29 Plaza (1989) gave the following two extra introspection axioms on top of $\mathbb{S} 5$ to capture this announcement-free fragment without a proof: $K v_{i} c \rightarrow K_{i} K v_{i} c$ and $\neg K v_{i} c \rightarrow K_{i} \neg K v_{i} c$. Our later language will supersede this simple language.

${ }^{30}$ For example, I know that I have hands given that I am not a brain in a vat. 


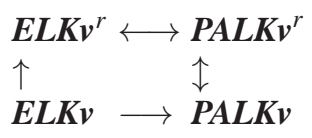

It means that we can forget about PALKv and use $\mathbf{E} \mathbf{L} \mathbf{K v}^{r}$ instead, qua expressivity.

\subsubsection{Axiomatization}

An axiomatization for the multi-agent $\mathbf{E} \mathbf{L} \mathbf{K v}^{r}$ is given by Wang and Fan (2014): System $\mathbb{S E L I K V} \mathbb{V}^{r} \mathbb{S} 5$

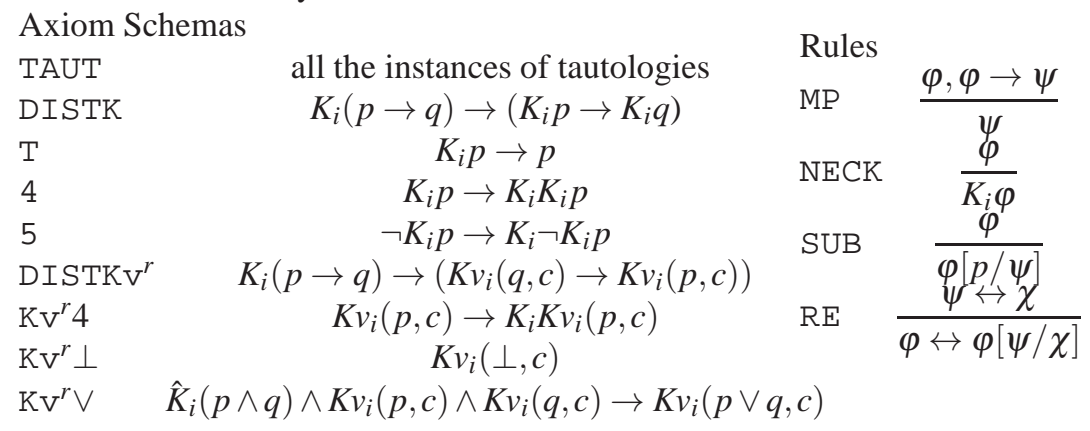

where DISTKV ${ }^{r}$ is the distribution axiom for the conditional $K v_{i}$ operator, which capture the interaction between $K_{i}$ and $K v_{i}$ (note the positions of $p$ and $q$ in the consequent). $\mathrm{Kv}^{r} 4$ is a variation of the positive introspection axiom, and the corresponding negative introspection is derivable. $\mathrm{Kv}^{r} \perp$ stipulates that the $K v_{i}$ operator is essentially a conditional. Maybe the most interesting axiom is $\mathrm{Kv}^{r} \vee$ which handles the composition of the conditions: suppose all the epistemically possible $p$-worlds agree on what $c$ is and all the epistemically possible $q$-worlds also agree on $c$, then the overlap between $p$-possibilities and $q$-possibilities implies that all the $p \vee q$ possibilities also agree on what $c$ is. The careful reader may spot similarity between this axiom and the formula to show incompleteness in Theorem 5 .

Wang and Fan (2014) then showed the completeness of the above system:

Theorem 7. SELIKV $\mathbb{V}^{r}$ is sound and strongly complete for $\boldsymbol{E} \boldsymbol{L K} \boldsymbol{v}^{r}$.

The highly non-trivial proof of the above theorem demonstrates the asymmetry between the syntax and semantics that we mentioned earlier. First note that in the canonical model, merely maximal consistent sets cannot work. The following is a model where two logically equivalent states are needed to falsify $K v_{1} c$, where $c$ is assigned value $\circ$ and $\bullet$ respectively. This can never be embedded into a canonical model where states are maximal consistent sets. This problem is due to the fact that our language is too weak to capture all the information in the models.

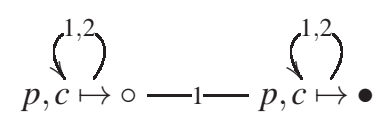


The proof idea comes when we realize what those $K v_{i}(\varphi, c)$ formulas actually are. Here, the perspective of quantified epistemic logic helps. Essentially, $K v_{i}(\varphi, c)$ can be viewed as $\exists x K_{i}(\varphi \rightarrow c=x)$ where $x$ is a variable and $c$ is a non-rigid constant. The $K v_{i}$ operator packages a quantifier, a modality, an implication and an equality together, without allowing the subformulas to appear freely. To build a suitable canonical model, we need to saturate each maximal consistent set with some extra information which roughly correspond to some subformulas of $\exists x K_{i}(\varphi \rightarrow c=x)$ :

- counterparts of atomic formulas such as $c=x$;

- counterparts of $K_{i}(\varphi \rightarrow c=x)$.

Moreover, we need to make sure these extra pieces of information are "consistent" with the maximal consistent sets and the canonical relations, by imposing further conditions. Wang and Fan (2014) introduced two functions $f$ and $g$ to tell the current value of each $c$, and the potential value of $c$ given $\varphi$ according to $i$. Thus a state in the canonical model is a triple $\langle\Gamma, f, g\rangle$ where $f$ and $g$ function as subformulas $c=x$ and $K_{i}(\varphi \rightarrow c=x)$. The extra conditions need to impose the consistency between such "subformulas" and the corresponding maximal consistent sets, e.g., $\psi \wedge K v_{i}(\psi, c) \in \Gamma$ implies $f(c)=g(i, \psi, c)$ : if $\psi$ holds on the current world, then the value of $c$ given $\psi$ should be the same as the current value of $c$.

Then we can prove the following statements:

- Each maximal consistent set can be properly saturated with some $f$ and $g$.

- Each saturated MCS including $\neg K_{i} \neg \varphi$ has a saturated $\varphi$-successor.

- Each saturated MCS including $\neg K v_{i}(\varphi, c)$ has two saturated $\varphi$-successors which disagree on the value of $c$.

As in the case of knowing whether, the last "existence lemma" requires us to build two successors simultaneously based on some consistent sets, where axiom $\mathrm{Kv}^{r} \vee: \hat{K}_{i}(p \wedge q) \wedge K v_{i}(p, c) \wedge K v_{i}(q, c) \rightarrow K v_{i}(p \vee q, c)$ plays an important role. See Wang and Fan (2014) for details.

Coming back to the original question by Plaza, we can now axiomatize multiagent PALKv ${ }^{r}$ by adding the following reduction axiom schemas easily 31

$$
\begin{array}{lcl}
\text { ! ATOM } & \langle\psi\rangle p & \leftrightarrow(\psi \wedge p) \\
\text { ! NEG } & \langle\psi\rangle \neg \varphi \leftrightarrow(\psi \wedge \neg\langle\psi\rangle \varphi) \\
\text { ! CON } & \langle\psi\rangle(\varphi \wedge \chi) \leftrightarrow(\langle\psi\rangle \varphi \wedge\langle\psi\rangle \chi) \\
\text { ! K } & \langle\psi\rangle K_{i} \varphi \leftrightarrow\left(\psi \wedge K_{i}(\psi \rightarrow\langle\psi\rangle \varphi)\right) \\
\text { ! } \mathrm{Kv}^{r} & \langle\varphi\rangle K v_{i}(\psi, c) \leftrightarrow\left(\varphi \wedge K v_{i}(\langle\varphi\rangle \psi, c)\right)
\end{array}
$$

Note that the specific values do not show in the language, and this gives us the hope to build models with a small domain and a small set of possible worlds for each satisfiable $\mathbf{E} \mathbf{L} \mathbf{K v}^{r}$ formulas. It can be shown that $\mathbf{E} \mathbf{L} \mathbf{K v}^{r}$ is not only decidable but with a complexity not higher than standard modal logic 3

Theorem 8 (Ding (2015)). ELK $\boldsymbol{v}^{r}$ over arbitrary models is PSPACE-complete.

${ }^{31}$ Uniform substitution does not work for these new schemas.

32 The decidability of $\mathbf{E} \mathbf{L} \mathbf{K v}^{r}$ over epistemic models was shown by Xiong (2014). 


\subsubsection{Simplification of the semantics}

As we mentioned, the models for $\mathbf{E} \mathbf{L} \mathbf{K v}^{r}$ are rich, but the language is quite weak, thus some information in the model cannot be expressed. To restore the symmetry between semantics and syntax, we may try to simplify the models while keeping the same logic intact (valid formulas). As we will see, the simplified semantics may sharpen our understanding of the logic and facilitate further technical discussions.

Let us start with a simple but crucial observation that we already touched implicitly in the discussion of the completeness proof: $\neg K v_{i}(\varphi, c)$ can be viewed as a special diamond formula, since it says that there are two $i$-accessible $\varphi$-worlds that do not agree on the value of $c 33$ Note that the semantics does not really rely on the exact value of $c$ on each world, but it does depend on whether $c$ has the same value. This inspires Gu and Wang (2016) to propose a simplified semantics, which interprets the corresponding diamond $\diamond_{i}^{c}$ w.r.t. a ternary relation $R_{i}^{c}$ in the Kripke models, where $s R_{i}^{c} u v$ intuitively means that $u, v$ are two $i$-successors of $s$, which do not agree on the value of $c 34$

Let us consider the following language $\mathbf{M L K v}{ }^{r}$ (essentially a disguised rewritten version of $\mathbf{E L K} \mathbf{v}^{r}$ by replacing $K_{i}$ with $\square_{i}$, and $\neg K v_{i}$ with $\diamond_{i}^{c}$ )

$$
\varphi::=\top|p| \neg \varphi|(\varphi \wedge \varphi)| \square_{i} \varphi \mid \diamond_{i}^{c} \varphi
$$

The models are propositional Kripke models with both binary and ternary relations $\left\langle S,\left\{\rightarrow_{i}: i \in \mathbf{I}\right\},\left\{R_{i}^{c}: i \in \mathbf{I}, c \in \mathbf{C}\right\}, V\right\rangle$, where $\rightarrow_{i}$ is as before for the $\square_{i}$ operator. To simplify discussions, we do not assume $\rightarrow_{i}$ to be an equivalence relation in this subsection. The semantics for $\diamond_{i}^{c} \varphi$ is as follows:

$$
\mathscr{M}, s \vDash \diamond_{i}^{c} \varphi \Longleftrightarrow \exists u, v: \text { such that } s R_{i}^{c} u v, \mathscr{M}, u \vDash \varphi \text { and } \mathscr{M}, v \vDash \varphi
$$

To maintain the same logic (valid formulas modulo the rewriting), the following three conditions on $R_{i}^{c}$ are imposed.

1. Symmetry: $s R_{i}^{c} v u$ iff $s R_{i}^{c} u v$;

2. Inclusion: $s R_{i}^{c} u v$ only if $s \rightarrow_{i} u$ and $s \rightarrow_{i} v$;

3. Anti-Euclidean property: $s R_{i}^{c} t_{1} t_{2}$ and $s \rightarrow_{i} u$ implies that at least one of $s R_{i}^{c} u t_{1}$ and $s R_{i}^{c} u t_{2}$ holds.

The first two conditions are intuitive, given the intention of $R_{i}^{c}$. The condition (3) is the most interesting one and it is depicted as follows:

\footnotetext{
${ }^{33}$ In some applications in computer science, the exact value is also not that important, but people care about whether two values are equivalent, e.g., see logic works on data words (Bojańczyk et al, 2011; Boiańczyk, 2013). The author thanks Martin Otto for pointing this out.

${ }^{34}$ Instead of the ternary relation, it seems also natural to introduce an anti-equivalence relation $R^{c}$ such that $s R^{c} t$ intuitively means that $s$ and $t$ do not agree on the value of $c$. However, this approach faces troubles due to the limited expressive power of the modal language, see Gu and Wang, 2016) for a detailed discussion.
} 

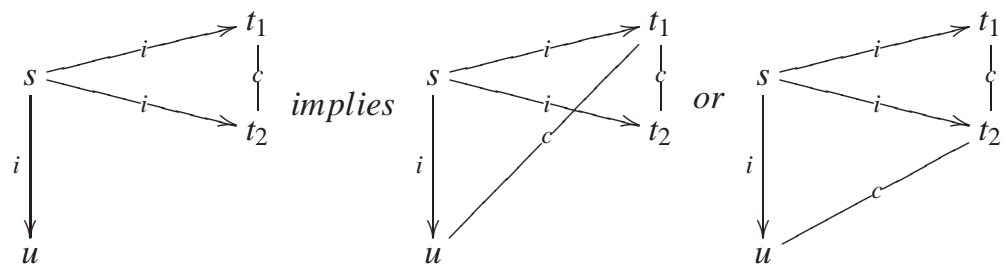

It says that if two $i$-accessible worlds do not agree on the value of $c$ then any third $i$-accessible world must disagree with one of the two worlds on $c$.

Given a first-order Kripke model for $\mathbf{E} \mathbf{L} \mathbf{K v}^{r}$, we have a corresponding Kripke model with both binary and ternary relations for $\mathbf{M L K \mathbf { v } ^ { r }}$, by defining $R_{i}^{c}$ as $\{(s, u, v) \mid$ $s \rightarrow_{i} u, s \rightarrow_{i} v$, and $\left.V_{\mathbf{C}}(c, u) \neq V_{\mathbf{C}}(c, v)\right\}$. Such a computed relation satisfy the above three properties ${ }^{35}$ Moreover, Gu and Wang (2016) show that the following proof system (essentially the translated version of $\mathrm{S} 5$-free $\mathbb{S E L} \mathbb{K} \mathbb{V}^{r}$ ) is sound and strongly complete w.r.t. the Kripke models satisfying (1) - (3).

Another look of $\mathbb{S E L} \mathbb{K} \mathbb{V}^{r}$

$$
\begin{aligned}
& \text { Axiom Schemas } \\
& \begin{array}{lc}
\text { TAUT } & \text { all the instances of tautologies } \\
\text { DISTK } & \square_{i}(p \rightarrow q) \rightarrow\left(\square_{i} p \rightarrow \square_{i} q\right) \\
\text { DISTKv }^{r} & \square_{i}(p \rightarrow q) \rightarrow\left(\diamond_{i}^{c} p \rightarrow \diamond_{i}^{c} q\right) \\
\mathrm{Kv}^{r} \perp & \quad \neg \diamond_{i}^{c} \perp \\
\mathrm{Kv}^{r} \vee & \diamond_{i}(p \wedge q) \wedge \diamond_{i}^{c}(p \vee q) \rightarrow\left(\diamond_{i}^{c} p \vee \diamond_{i}^{c} q\right)
\end{array}
\end{aligned}
$$$$
\begin{array}{ll}
\text { Rules } & \\
\text { MP } & \frac{\varphi, \varphi \rightarrow \psi}{\psi} \\
\text { NECK } & \frac{\varphi}{\square_{i} \varphi} \\
\text { SUB } & \frac{\varphi}{\varphi[p / \psi]} \\
\text { RE } & \frac{\psi \leftrightarrow \chi}{\varphi \leftrightarrow \varphi[\psi / \chi]}
\end{array}
$$

We can massage the system into an equivalent form to make it look more familiar by adding the necessitation rule $\mathrm{NECKv}^{r}$, deleting the $\mathrm{Kv}^{r} \perp$, and changing the shape of DISTKv (see (Gu and Wang, 2016) for the proof of equivalence):

$$
\begin{aligned}
& \text { Massaged } \mathbb{S E L} \mathbb{K} \mathbb{V}^{r} \\
& \text { Axiom Schemas } \\
& \text { TAUT all the instances of tautologies } \\
& \text { DISTK } \quad \square_{i}(p \rightarrow q) \rightarrow\left(\square_{i} p \rightarrow \square_{i} q\right) \\
& \operatorname{DISTKV}^{r} \quad \square_{i}(p \rightarrow q) \rightarrow\left(\square_{i}^{c} p \rightarrow \square_{i}^{c} q\right) \\
& \mathrm{KV}^{r} \vee \quad \diamond_{i}(p \wedge q) \wedge \diamond_{i}^{c}(p \vee q) \rightarrow\left(\diamond_{i}^{c} p \vee \diamond_{i}^{c} q\right) \\
& \begin{array}{lc}
\text { Rules } & \multicolumn{1}{c}{\text { MP }} \\
\text { NECK } & \frac{\varphi, \varphi}{\psi} \\
\text { NECKV } & \frac{\varphi}{\square_{i} \varphi} \\
\text { SUB } & \frac{\square_{\dot{\varphi}}^{c} \varphi}{\varphi[p / \psi]} \\
\text { RE } & \frac{\psi \leftrightarrow \chi}{\varphi \leftrightarrow \varphi[\psi / \chi]}
\end{array}
\end{aligned}
$$

It seems that $\square_{i}^{c}$ (the dual of $\diamond_{i}^{c}$ ) almost behaves just like a normal modality. However, the distribution axiom $\square_{i}^{c}(p \rightarrow q) \rightarrow\left(\square_{i}^{c} p \rightarrow \square_{i}^{c} q\right)$ is not valid. This is because $\diamond_{i}^{c}$ is essentially a binary diamond, but we force the two arguments to be the same! To restore the normality, we can consider the following language: $\left(\mathbf{M L K v} \mathbf{v}^{b}\right)$ :

35 Clearly the corresponding models also satisfy more properties, such as $s R_{i}^{c} u v$ only if $v \neq u$. However, (1)-(3) are enough to keep the logic intact, see (Gu and Wang, 2016) for details. 


$$
\varphi::=\top|p| \neg \varphi|(\varphi \wedge \varphi)| \square_{i} \varphi \mid \diamond_{i}^{c}(\varphi, \varphi)
$$

which allow formulas $\diamond_{i}^{c}(\varphi, \psi)$ where $\varphi \neq \psi . \diamond_{i}^{c}(\varphi, \psi)$ intuitively says that there are two $i$-successors such that one satisfies $\varphi$ and the other satisfies $\psi$ and they do not agree on the value of $c$. The semantics is now standard for a binary modality:

$$
\mathscr{M}, s \vDash \diamond_{i}^{c}(\varphi, \psi) \Longleftrightarrow \exists u, v: \text { such that } s R_{i}^{c} u v, \mathscr{M}, u \vDash \varphi \text { and } \mathscr{M}, v \vDash \psi
$$

Surprisingly, the above language $\mathbf{M L K v} \mathbf{v}^{b}$ is equally expressive as $\mathbf{M L K v} \mathbf{v}^{r}$ under the key observation by Gu and Wang (2016) that $\diamond_{i}^{c}(\varphi, \psi)$ is equivalent to the to the disjunction of the following three formulas:

1. $\diamond_{i}^{c} \varphi \wedge \diamond_{i} \psi$

2. $\diamond_{i}^{c} \psi \wedge \diamond_{i} \varphi$

3. $\diamond_{i} \varphi \wedge \diamond_{i} \psi \wedge \neg \diamond_{i}^{c} \varphi \wedge \neg \diamond_{i}^{c} \psi \wedge \diamond_{i}^{c}(\varphi \vee \psi)$

Now it is clear that $\mathbf{M L K v} \mathbf{v}^{b}$ over Kripke models with binary and ternary relations is just a normal modal logic, which also means that $\mathbf{M L K v} \mathbf{v}^{r}$ (and thus $\mathbf{E} \mathbf{L} \mathbf{K} \mathbf{v}^{r}$ ) can be viewed as a disguised normal modal logic qua expressivity. Now the axiomatization and other technical issues can be largely simplified by using standard techniques. Gu and Wang (2016) showed the completeness of the following normal modal logic system using standard techniques 36 where SYM, INC and ATEUC capture exactly the three properties respectively 37

$$
\text { System } \mathbb{S M L} \mathbb{K} \mathbb{V}^{b}
$$

$$
\begin{aligned}
& \text { Axiom Schemas } \\
& \text { TAUT all the instances of tautologies } \\
& \text { DISTK } \quad \square_{i}(p \rightarrow q) \rightarrow\left(\square_{i} p \rightarrow \square_{i} q\right) \\
& \operatorname{DISTKv}^{b} \square_{i}^{c}(p \rightarrow q, r) \rightarrow\left(\square_{i}^{c}(p, r) \rightarrow \square_{i}^{c}(q, r)\right) \\
& \text { SYM } \quad \square_{i}^{c}(p, q) \rightarrow \square_{i}^{c}(q, p) \\
& \text { INC } \quad \diamond_{i}^{c}(p, q) \rightarrow \diamond_{i} p \\
& \text { ATEUC } \quad \diamond_{i}^{c}(p, q) \wedge \diamond_{i} r \rightarrow \diamond_{i}^{c}(p, r) \vee \diamond_{i}^{c}(q, r)
\end{aligned}
$$

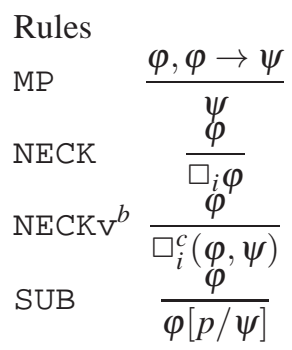

This normal modal logic view also gives us a standard bisimulation notion for $\mathbf{M L K v}^{b}$ on models with ternary and binary relations (cf. e.g., Blackburn et al (2002)). Then we can translate the bisimulation conditions on $R_{i}^{c}$ back to the conditions on $\rightarrow_{i}$ and the value assignment $V_{\mathbf{C}}$ to obtain a notion of bisimulation in the setting of FO epistemic models for $\mathbf{E} \mathbf{L} \mathbf{K v}^{r}$. As another potential application, we believe that the normal modal logic view can also shed some light on the decision procedure of $\mathbf{E L K v}{ }^{r}$, since the models of $\mathbf{M L K v}{ }^{r}$ are free of value assignments, which are much easier to handle.

\footnotetext{
${ }^{36}$ Note that here the maximal consistent sets are enough to build the canonical model due to the change of models, compared to canonical model for $\mathbf{E} \mathbf{L K} \mathbf{v}^{r}$.

${ }^{37}$ Due to SYM, we only need DISTKv ${ }^{b}$ and $\mathrm{NECKV}^{b}$ w.r.t. the first argument.
} 


\subsubsection{A new update operator}

We close the discussion on knowing value logic by another natural extension, which brings a surprising connection to dependence logic. So far, the updates we have considered are mainly public announcements. However, such updates are most suitable for changing knowledge-that. Actually, the knowing value operator $K v_{i}$ has also a very natural corresponding update operation. Gattinger et al (2017) enrich $\mathbf{E L K \mathbf { v } ^ { r }}$ with the public inspection operator $[c]$ (call the following language PILKv ${ }^{r}$ ):

$$
\varphi::=\top|p| \neg \varphi|(\varphi \wedge \varphi)| K_{i} \varphi\left|K v_{i}(\varphi, c)\right|[c] \varphi
$$

Intuitively, $[c] \varphi$ says that after revealing the actual value of $c, \varphi$ holds. It can be viewed as the knowing value analog of the public announcement of a formula. Formally, the semantics of $[c] \varphi$ is defined on first-order epistemic models $\mathscr{M}=\left\langle S, D,\left\{\sim_{i} \mid i \in \mathbf{I}\right\}, V, V_{\mathbf{C}}\right\rangle$ as follows:

$$
\mathscr{M},\left.s \vDash[c] \varphi \Leftrightarrow \mathscr{M}\right|_{c} ^{s}, s \vDash \varphi
$$

where $\left.\mathscr{M}\right|_{c} ^{s}=\left\langle S^{\prime}, D,\left\{\sim_{i}\left|S_{S^{\prime} \times S^{\prime}}\right| i \in \mathbf{I}\right\},\left.V\right|_{S^{\prime}},\left.V_{\mathbf{C}}\right|_{\mathbf{C} \times S^{\prime}}\right\rangle$ where $S^{\prime}=\left\{s^{\prime} \mid V_{\mathbf{C}}\left(c, s^{\prime}\right)=\right.$ $\left.V_{\mathbf{C}}(c, s)\right\}$ i.e., the update deletes the worlds which do not agree with the current world $s$ on the value of $c$. In contrast to the update of public announcement, the update here is local in the sense that the $s$ matters in the updated model $\left.\mathscr{M}\right|_{c} ^{s}$.

By adapting some suitable bisimulation notion, Gattinger et al (2017) showed that $\mathbf{P I L K v}{ }^{r}$ is more expressive than $\mathbf{E L K v}{ }^{r}$, thus $[c]$ is not reducible. Intuitively, the update $[c]$ may bring new information that is not pre-encoded by a formula.

Now with this new dynamic operator at hand, we can express the knowledge of dependence between different constants as $K d_{i}(c, d):=K_{i}[c] K v_{i} d . K d_{i}(c, d)$ intuitively says that agent $i$ knows that the value of $d$ depends on the value of $c$. Formally the semantics can be spelled out:

$$
\mathscr{M}, s \vDash K d_{i}(c, d) \Leftrightarrow \text { for all } t_{1} \sim_{i} s, t_{2} \sim_{i} s: t_{1}={ }_{c} t_{2} \Longrightarrow t_{1}={ }_{d} t_{2}
$$

where $t={ }_{c} t^{\prime}$ iff $V_{\mathbf{C}}(c, t)=V_{\mathbf{C}}\left(c, t^{\prime}\right)$. It is not hard to see that $K d_{i}(c, d) \wedge K v_{i}(\varphi, c) \rightarrow$ $K v_{i}(\varphi, d)$ is valid: knowing the dependence helps to know the value. Moreover, we can handle the knowledge of dependence between sets of constants. Given any two finite sets $D, E \subseteq \mathbf{C}$ such that $D=\left\{d_{1}, \ldots, d_{n}\right\}$ and $E=\left\{e_{1}, \ldots e_{m}\right\}$, let $K d_{i}(D, E):=$ $K_{i}\left[d_{1}\right] \ldots\left[d_{n}\right]\left(K v_{i} e_{1} \wedge \ldots \wedge K v_{i} e_{m}\right)$. Note that the order of public inspections does not really matter 38

$K d_{i}(c, d)$ can be viewed as the atomic formula $=(c, d)$ in dependence logic proposed by Väänänen (2007), w.r.t. the "team" model which consists of the $i$ accessible worlds (as value assignments for constants in $\mathbf{C}$ ). Note that there is a crucial difference between our approach and the team semantics of dependence atoms

\footnotetext{
${ }^{38}$ A similar operator with propositional arguments was proposed by Goranko and Kuusisto (2015) in the setting of knowing whether, which can express that given the truth values of $\varphi_{1}, \ldots, \varphi_{n}$ the agent $i$ knows whether $\varphi$.
} 
in dependence logic. We can specify the local dependence by $[c] K v_{i} d$ i.e., $i$ knows the value of $d$ given the actual value of $c$, whereas $=(c, d)$ can only specify global dependence as the distinction between $K_{i}[c] K v_{i} d$ and $[c] K v_{i} d$ shows. The connection with dependence logic also bring PILKv ${ }^{r}$ closer to the first-order variant of the epistemic inquisitive logic by Ciardelli and Roelofsen (2015), where the knowledge of entailment of interrogatives can also be viewed as our $K d_{i}(c, d)$. More precisely, $K d_{i}(c, d)$ can be expressed by $K_{i}(\bar{\exists} x(x=c) \rightarrow \bar{\exists} x(x=d))$, where $\bar{\exists}$ is the inquisitive existential quantifier and $\bar{\exists} x(x=c)$ corresponds to the question on the value of $c$. Intuitively, $K_{i}(\bar{\exists} x(x=c) \rightarrow \bar{\exists} x(x=d))$ says that agent $i$ knows that the answer to the question "what is $c$ ?" will determine the answer to the question "what is $d$ ?", see (Ciardelli, 2016, Sec. 6.7.4) for a detailed comparison with our approach.

Gattinger et al (2017) axiomatized the following single-agent fragment of PILKv $\mathbf{v}^{r}$ which can be considered as the $K v$ counterpart of the public announcement logic:

$$
\varphi::=\top|\neg \varphi| \varphi \wedge \varphi|K v c|[c] \varphi
$$

However the axiomatization of the full PILKv ${ }^{r}$ is still open. On the other hand, Baltag (2016) proposed a very general language with a similar conditional operator $K_{i}^{\left\{t_{1}, \ldots, t_{n}\right\}}$ where $t_{i}$ are terms that can contain function symbols over variables and formulas. $K_{i}^{\left\{t_{1}, \ldots, t_{n}\right\}} t$ says that $i$ knows the value of $t$ if he or she is given the values of $t_{1}, \ldots, t_{n}$. A distinct feature of this language, compared to the $K v$-based languages, is that it also includes equalities of terms as atomic formulas in order to obtain a complete axiomatization. It is shown that this language can pre-encode the public inspection operators and it is decidable.

\subsection{Knowing how}

Last but not the least, we will look at a logic of a particular kind of knowing how proposed and studied by Wang (2015a) 39 Compared to the previous two cases, it has a couple of special features worth mentioning:

- There is no consensus on the logical language and the semantics of the logic of knowing how.

- As we will see, although the knowing how formulas still follow roughly the general shape of $\exists x \square \varphi(x)$, the existential quantifier is not really a first-order one.

- Contrary to the previous cases of knowing whether and knowing what, there can be more than one $x$ that can make $\square \varphi(x)$ true in the knowing how case, and this requires new techniques in the completeness proof.

- Our model is no longer based on epistemic models with epistemic relations.

\footnotetext{
${ }^{39}$ The extended full version will appear as Wang (2017).
} 
Knowing how is frequently discussed in epistemology and in $\mathrm{AI} 40$ Philosophers debate about whether knowledge-how, the knowledge expressed by the knowing how expressions, can be reduced to knowledge-that, i.e. propositional knowledge 41 There are two major philosophical stances: intellectualists think knowledge-how is reducible to knowledge-that (cf. e.g., Stanley and Williamson (2001)), while antiintellectualists holds the opposite position that knowledge-how is irreducible (cf e.g., Ryle (1949)). At the first glance, knowing how seems to express a statement about ability, e.g., "I know how to swim" roughly says that I have the ability to swim. However, philosophy literature provides ample examples to show that this simple-minded idea is shaky, e.g., can you say you know how to digest food since you have that ability? As another example, in some cases even though you do not have the ability at the moment, it is still reasonable to claim the knowledge-how, e.g., a pianist with a broken-arm may still say he or she knows how to play piano, although due to the accident he or she cannot do it right now 42 Here the relevant insight is that knowing how expressions may come with implicit conditions. When we say that a chef knows how to cook Chinese dishes, it does not mean that he can do it right now, but it means he can do it given all the ingredients and facilities. Thus in the formal language we introduce a binary modality $K h(\psi, \varphi)$ meaning that I know how to achieve $\varphi$ given $\psi$. Note that $\psi$ may be false currently but we should look at all the worlds where it is true.

In AI, ever since the pioneering works by McCarthy (1979) and Moore (1977), formalizing the interaction of knowledge and ability has been an important issue till now (cf. (Gochet, 2013; $\AA$ @gotnes et al, 2015) for up-to-date overviews). One problem that logicians in AI face is that simply combining "knowing that" and "ability" does not lead to a natural notion of knowing how, as sharply pointed out by Herzig (2015). For example, adding the knowing-that operator to alternating temporal logic (ATL) can result in a logic which can express one knows that there is a strategy to achieve some goal, which is in the de dicto shape of $K \exists x \varphi(x)$ rather than the desired de re shape $\exists x K \varphi(x)$. We need a way to somehow insert the $K$ modality in-between the implicit existential quantifier and the strategy modality ${ }^{43}$ We tackle this problem by packing the quantifier and the modality together in the $K h$ operator with a semantics inspired by conformant planning in AI, where the goal is to find a uniform plan (action sequence) such that at all the initial situations the plan will always work and reach the goal (cf. Yu et al (2016)). Knowing how to achieve $\varphi$ given $\psi$ then amounts to having a conformant plan which works for all the $\psi$-worlds.

\footnotetext{
${ }^{40}$ See (Wang, 2015a) for a more detailed survey.

41 See the collection of papers on the topic at philpaper edited by by John Bengson: http://philpapers.org/browse/knowledge-how

${ }^{42}$ Such examples motivated intellectualists to propose an account other than treating knowledgehow simply as ability. A notable approach proposed by Stanley and Williamson (2001) breaks down "knowing how to $F$ " into: "There is a way such that I know it is a way to do $\mathrm{F}$, and I entertain it in a practical mode of presentation." Note that it essentially has the familiar shape $\exists x K \varphi(x)$, which also inspired the semi-formal treatment by Lau and Wang (2016).

${ }^{43}$ See (Herzig, 2015) for some existing solutions, e.g. by using epistemic STIT logic proposed by Broersen and Herzig (2015).
} 
Before going into the details, some clarifications have to be made.

- We only focus on goal-directed knowing-how, as Gochet (2013) puts it, e.g., knowing how to prove a theorem, how to open the door, how to bake a cake, and how to cure the disease.

- We do not study knowing-how in the following senses: I know how the computer works (explanation); I know how happy she is (degree of emotion); I know how to behave at the dinner table (rule-directed).

\subsubsection{Language and semantics}

As inspired by the philosophy literature, we introduce a conditional knowing-how operator in the following single-agent language $\mathbf{L}_{\mathbf{K h}}$ Wang, 2015a):

$$
\varphi \quad::=\top|p| \neg \varphi|(\varphi \wedge \varphi)| \operatorname{Kh}(\varphi, \varphi)
$$

Intuitively, $K h(\psi, \varphi)$ says that the agent knows how to achieve $\varphi$ given the condition $\psi . U \varphi$ is defined as $K h(\neg \varphi, \perp)$, which is intended to be a universal modality to be explained later.

Given a non-empty set of propositional letters $\mathbf{P}$, a non-empty set of actions $\mathbf{A}$, a model is simply a tuple $(S, R, V)$ where:

- $S$ is a non-empty set of states;

- $R: \mathbf{A} \rightarrow 2^{S \times S}$ is a collection of transitions labelled by actions in $\mathbf{A}$;

- $V: S \rightarrow 2^{\mathbf{P}}$ is a valuation function.

Note that this is not a standard epistemic model for there is no epistemic alternative relation in the model. Intuitively, the model represents the ability that the agent has, and it can be used as a model for an epistemic logic of knowing how (cf. also Wang, 2015b) for a more general setting.). For example, the left model below represents that the agent can do $a$ on $s_{1}$ but he cannot control the outcome. On the other hand he can do $b$ on $s_{2}$ which leads to a single $q$-world.

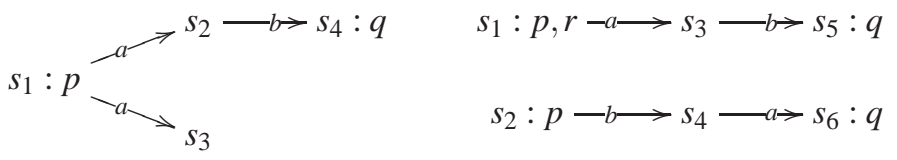

Intuitively, given only $p$, the agent should not know how to reach $q$ in the above two models: although $a b$ leads to $q$ in the left model, $a$ cannot control the result of $a$; he may fail to continue to do $b$ after doing $a$. For the right model, although the agent can do $a b$ to reach $q$ on $s_{1}$ and do $b a$ to reach $q$ on $s_{2}$, he does not know where he is exactly given only $p$, and thus does not have a uniform plan which can always work. We flesh out such intuition in the following semantics:

$\begin{aligned} \mathscr{M}, s \vDash K h(\psi, \varphi) \Leftrightarrow & \text { there exists an action sequence } \sigma \in \mathbf{A}^{*} \text { such that for all } \mathscr{M}, s^{\prime} \vDash \psi: \\ & \text { (1) } \sigma \text { is strongly executable at } s^{\prime}, \text { and } \\ & \text { (2) for all } t \text { if } s^{\prime} \stackrel{\sigma}{\rightarrow} t \text { then } \mathscr{M}, t \vDash \varphi\end{aligned}$


where $\sigma=a_{1} \ldots a_{n}$ is strongly executable at $s^{\prime}$ if $s^{\prime}$ has at least one $a_{1}$-successor and for any $1 \leq k<n$ and any $t, s^{\prime} \stackrel{a_{1} \ldots a_{k}}{\rightarrow} t$ implies that $t$ has at least one $a_{k+1}$-successor. Intuitively, $\sigma$ is strongly executable iff $\sigma$ is executable and whenever you start doing an initial segment of $\sigma$, you can always continue. For example $a b$ is not strongly executable at $s_{1}$ in the left model above, since it may fail. Note that the quantifier schema in the semantics is $\exists \forall$ which is in compliance with the general schema $\exists x K$, although now the existential quantifier is no longer first-order, and the $K$ is replaced by a quantifier induced by the condition $\psi$ representing the initial uncertainty.

One can verify that $s_{1} \vDash \neg K h(p, q)$ in the above two models and $s_{1} \vDash K h(p, q)$ in the model below, since there is a strongly executable plan $r u$ from any $p$-world to some $q$-world.

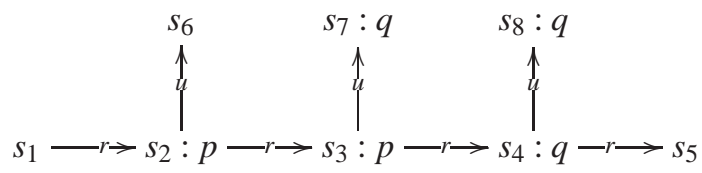

Now it can also be verified that $U$ is indeed a universal modality:

$\mathscr{M}, s \vDash U \varphi \Leftrightarrow K h(\neg \varphi, \perp) \Leftrightarrow$ for all $t \in \mathscr{S}, \mathscr{M}, t \vDash \varphi$

\subsubsection{Axiomatization}

A complete axiomatization is given in (Wang, 2015a) using $K h$ and the definable $U$ :

$$
\begin{aligned}
& \text { System } \mathbb{S K K H} \\
& U(p \rightarrow q) \rightarrow K h(p, q) \\
& \frac{\varphi, \varphi \rightarrow \psi}{\frac{\varphi}{U \varphi}} \\
& \frac{\varphi(p)}{\varphi[\psi / p]} \\
& U p \rightarrow p \\
& K h(p, q) \rightarrow U K h(p, q) \\
& \neg K h(p, q) \rightarrow U \neg K h(p, q)
\end{aligned}
$$

We can view $U$ as a knowing that operator for the background knowledge taken for granted in the model, and it indeed behaves as an $\$ 5$ modality $445 \mathrm{KU}$ and $4 \mathrm{KU}$ are the introspection axioms. EMP says that if you know $p$ implies $q$ then you trivially

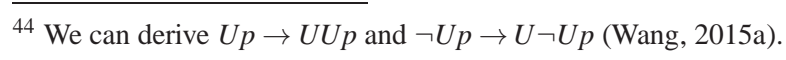


know how to achieve $q$ given $p$, i.e., doing nothing. The most interesting axiom is COMPKh, which says knowledge-how can be sequentially composed. Moreover, two interesting axioms below can be derived from the above system. WSKh says that you can strengthen the precondition and weaken the goal and still know how; POSTKh is a recursive way of expressing the compositionality of knowing-how.

\begin{tabular}{|c|c|}
\hline WSKh & $U(p \rightarrow r) \wedge U(o \rightarrow q) \wedge K h(r, o) \rightarrow K h(p, q)$ \\
POSTKh & $K h(r, K h(p, q) \wedge p) \rightarrow K h(r, q)$ \\
\hline
\end{tabular}

Theorem 9 (Wang (2015a)). SKHE is sound and strongly complete w.r.t. the class of all models.

The completeness proof involves building special canonical models 45 where every $K h(\psi, \varphi)$ can be realized by a simple one-step simple plan. Note that in contrast with the previous logics of knowing whether and knowing value, when showing $\neg K h(\psi, \varphi)$ is true at a maximal consistent set including it, it is no longer enough to build two differentiating states, since the existential quantifier hidden in $K h$ no longer assumes uniqueness: there can be many plans to achieve $\varphi$ on a given $\psi$ world 46 However, you need to show no single plan will do the job uniformly over all the $\psi$-worlds.

In a canonical model, all the states share the same $K h$-formulas, it is then easy to prove that the size of the canonical model is bounded by $2^{n}$ where $n$ is the number of propositional letters. Therefore for a given $\mathbf{L}_{\mathbf{K h}}$ formula $\varphi$, if it is satisfiable then it is satisfiable in a model which is bounded by $2^{|\varphi|}$. This leads to the small model property of the logic, and the decidability follows since we have a finite axiomatization, as shown by Wang (2017).

It is also natural to generalize the $K h$ operator to a ternary one with an extra intermediate constraint. $K h(\psi, \chi, \varphi)$ then says that "the agent knows how to achieve $\varphi$ given $\psi$ while maintaining $\chi$ in-between." In this way we can handle knowledgehow with constants about the process of the plan. The logic of this ternary modality is formally characterized by Li and Wang (2017).

Having presented our examples of the logics of knowing whether, knowing what, and knowing how, we encourage the readers to go back to the summary of the highlights about each logic at the beginning of Section 4

\section{Conclusions and future work}

This paper advocates the study of epistemic logics of knowing-wh. We started with a survey on Hintikka's contributions to knowing-wh, and the relevant recent literature on quantified epistemic logic. Then we proposed a new approach to epistemic logics of knowing-wh, which takes each knowing-wh as a single modality. In this way we

\footnotetext{
${ }^{45}$ For each maximal consistent set we build a canonical model (Wang, 2015a).

${ }^{46}$ Recall that $\neg K v_{i} c$ is true if there are two states which disagree on $c$.
} 
can "hide" the quantifiers inside modalities, thus limiting the expressivity of the language in order to avoid conceptual and technical problems of the full quantified epistemic logic. By three example studies on knowing whether, knowing what and knowing how, we demonstrated the usefulness and the diversity of knowing-wh logics. We hope we have shown that this new approach may lead us to:

- interesting (non-normal) modal operators packaging a quantifier and a (standard) modality $(\exists x \square)$;

- new meaningful axioms about different knowing-wh and their interactions with the knowing that operator;

- discovery of computationally (relatively) cheap fragments of first-order or higherorder modal logics;

- interesting connections with existing logics;

- various techniques handling the completeness proof of such non-normal modal logics;

- techniques restoring the symmetry between a weak language and rich models.

In some sense, our approach is a minimalistic one. We do not have the ambition to fit everything about knowing-wh in a very powerful language with full compositionality and the flexibility to capture the context-sensitivity. Instead, we start from very simple languages of some particular knowing-wh constructions, fix some intuitive semantics which can account for some useful readings, and then see whether we can capture the decidable logics nicely. Essentially, we are following the successful story of propositional modal logic, which packages quantifiers and other constructions together in modalities. This minimalistic idea distinguishes us from the quantified epistemic logic approach by Hintikka and others, and the linguistically motivated inquisitive semantics approach to the logic of knowing-wh. Our examples also showed that although the hidden logical structures of various knowing-wh modalities may be similar to each other to some extent, the details of the language, models, and the semantics matter a lot in deciding the concrete axioms for different knowing-wh. The newly introduced modalities also let us see clearly the special features of different knowing-wh, which may not be possible if we break everything down into quantifiers, predicates, and standard modalities in a quantified epistemic logic.

Having said the above, we are also aware of the obvious limitations of our approach. Readers are encouraged to go back to Section 3 to review the discussion on the advantages and limitations of our approach. We think both the minimalistic approach and the "maximalistic" approaches are good for their own purposes, and the two approaches can be beneficial for each other by bringing new insights to balance expressive power and complexity further.

We believe this is only the beginning of an exciting story. Besides the epistemic logics of other types of knowing-wh such as knowing why (Xu et al, 2016) and knowing who and so on, there are a lot of general topics to be discussed about the existing logics mentioned in this paper. For example:

- model theory, proof theory, and complexity of the knowing-wh logics; 
- group notions of knowing-wh, e.g., commonly knowing whether, jointly knowing how and so on;

- new update mechanisms to change knowing-wh, e.g., learning new abilities in the model of knowing how;

- simplified semantics, e.g., new semantics of knowing how logic that can keep the valid axioms intact but restores the symmetry between syntax and semantics, as in the case of knowing value logic.

- alternative semantics, e.g., multi-agent, contingent planning based knowing how logic, where branching plans are used;

- logical omniscience of knowing-wh;

- the study of the generic modality which packs $\exists x \square$ together, and its connection to monodic and other decidable fragments of quantified modal logic.

This new generation of epistemic logics will open up various opportunities for epistemic logicians to explore 47

Acknowledgements The author acknowledges the support from the National Program for Special Support of Eminent Professionals and NSSF key projects 12\&ZD119. The author is grateful to Hans van Ditmarsch for his very detailed comments on an early version of this paper. The author also thanks the anonymous reviewer who gave many constructive suggestions including the observation in footnote 15

\section{References}

Ågotnes T, Goranko V, Jamroga W, Wooldridge M (2015) Knowledge and ability. In: van Ditmarsch H, Halpern J, van der Hoek W, Kooi B (eds) Handbook of Epistemic Logic, College Publications, chap 11, pp 543-589

Aloni M (2001) Quantification under conceptual covers. PhD thesis, University of Amsterdam

Aloni M (2016) Knowing-who in quantified epistemic logic. In: van Ditmarsch H, Sandu G (eds) Jaakko Hintikka on knowledge and game theoretical semantics, Springer

Aloni M, Roelofsen F (2011) Interpreting concealed questions. Linguistics and Philosophy 34(5):443-478

Aloni M, Égré P, de Jager T (2013) Knowing whether A or B. Synthese 190(14):2595-2621

Aumann R (1989) Notes on interactive epistemology. In: Cowles Foundation for Reaserch in Economics working paper

Baltag A (2016) To know is to know the value of a variable. In: Proceedings of AiML Vol. 11, pp 135-155

${ }^{47}$ In Hintikka's terms, maybe it can be called the 1.5th generation of epistemic logics, since it is not as general as Hintikka's idea of the second generation epistemic logics. 
Belardinelli F, van der Hoek W (2015) Epistemic quantified boolean logic: Expressiveness and completeness results. In: Proceedings of IJCAI '15, AAAI Press, pp 2748-2754

Belardinelli F, van der Hoek W (2016) A semantical analysis of second-order propositional modal logic. In: Proceedings of AAAI'16, pp 886-892

Belardinelli F, Lomuscio A (2009) Quantified epistemic logics for reasoning about knowledge in multi-agent systems. Artificial Intelligence 173(9-10):982-1013

Belardinelli F, Lomuscio A (2011) First-order linear-time epistemic logic with group knowledge: An axiomatisation of the monodic fragment. Fundamenta Informaticae 106(2-4):175-190

Belardinelli F, Lomuscio A (2012) Interactions between knowledge and time in a first-order logic for multi-agent systems: Completeness results. Journal of Artificial Intelligence Research 45:1-45

Blackburn P, de Rijke M, Venema Y (2002) Modal Logic. Cambridge University Press

Boer SE, Lycan WG (2003) Knowing Who. The MIT Press

Bojańczyk M (2013) Modelling infinite structures with atoms. In: Libkin L, Kohlenbach U, de Queiroz R (eds) Proceedings of WoLLIC'13, Springer, pp 13-28

Bojańczyk M, David C, Muscholl A, Schwentick T, Segoufin L (2011) Two-variable logic on data words. ACM Transactions on Computational Logic 12(4):27:127:26

Braüner T, Ghilardi S (2007) First-order modal logic. In: Blackburn P, van Benthem J, Wolter F (eds) Handbook of Modal Logic, pp 549-620

Broersen J, Herzig A (2015) Using STIT theory to talk about strategies. In: van Benthem J, Ghosh S, Verbrugge R (eds) Models of Strategic Reasoning: Logics, Games, and Communities, Springer, pp 137-173

Chaum D (1988) The dining cryptographers problem: Unconditional sender and recipient untraceability. Journal of Cryptology 1(1):65-75

Ciardelli I (2014) Modalities in the realm of questions: Axiomatizing inquisitive epistemic logic. In: Proceedings of AiML Vol. 10, pp 94-113

Ciardelli I (2016) Questions in logic. PhD thesis, University of Amsterdam

Ciardelli I, Roelofsen F (2015) Inquisitive dynamic epistemic logic. Synthese 192(6):1643-1687

Ciardelli I, Groenendijk J, Roelofsen F (2013) Inquisitive semantics: A new notion of meaning. Language and Linguistics Compass 7(9):459-476

Cohen M, Dam M (2007) A complete axiomatization of knowledge and cryptography. In: Proceedings of LiCS '07, IEEE Computer Society, pp 77-88

Corsi G (2002) A unified completeness theorem for quantified modal logics. Journal of Symbolic Logic 67(4):1483-1510

Corsi G, Orlandelli E (2013) Free quantified epistemic logics. Studia Logica 101(6):1159-1183

Corsi G, Tassi G (2014) A new approach to epistemic logic. In: Logic, Reasoning, and Rationality, Springer, pp 25-41

Cresswell MJ (1988) Necessity and contingency. Studia Logica 47(2):145-149 
Demri S (1997) A completeness proof for a logic with an alternative necessity operator. Studia Logica 58(1):99-112

Ding Y (2015) Axiomatization and complexity of modal logic with knowing-what operator on model class $\mathrm{K}$, URL http://www.voidprove.com/research.html, unpublished manuscript

van Ditmarsch H (2007) Comments to 'logics of public communications'. Synthese 158(2):181-187

van Ditmarsch H, Fan J (2016) Propositional quantication in logics of contingency. Journal of Applied Non-Classical Logics (forthcoming)

van Ditmarsch H, van der Hoek W, Kooi B (2007) Dynamic Epistemic Logic. Springer

van Ditmarsch H, van der Hoek W, Illiev P (2012a) Everything is knowable how to get to know whether a proposition is true. Theoria 78(2)

van Ditmarsch H, van der Hoek W, Kooi B (2012b) Local properties in modal logic. Artificial Intelligence 187-188:133 - 155

van Ditmarsch H, Fan J, van der Hoek W, Iliev P (2014) Some exponential lower bounds on formula-size in modal logic. In: Proceedings of AiML Vol.10, pp 139157

van Ditmarsch H, Halpern J, van der Hoek W, Kooi B (eds) (2015) Handbook of Epistemic Logic. College Publications

Egré P (2008) Question-embedding and factivity. Grazer Philosophische Studien 77(1):85-125

Fagin R, Halpern J, Moses Y, Vardi M (1995) Reasoning about knowledge. MIT Press, Cambridge, MA, USA

Fan J (2015) Logical studies for non-contingency operator. PhD thesis, Peking University, (in Chinese)

Fan J, van Ditmarsch H (2015) Neighborhood contingency logic. In: Proceedings of ICLA'15, pp 88-99

Fan J, Wang Y, van Ditmarsch H (2014) Almost neccessary. In: Proceedings of AiML Vol.10, pp 178-196

Fan J, Wang Y, van Ditmarsch H (2015) Contingency and knowing whether. The Review of Symbolic Logic 8:75-107

Fine K (1970) Propositional quantifiers in modal logic. Theoria 36(3):336-346

Fitch F (1963) A logical analysis of some value concepts. Journal of Symbolic Logic 28(2):135-142

Fitting M, Mendelsohn RL (1998) First-Order Modal Logic. Springer

van Fraassen B (1980) The scientific image. Oxford: Oxford University Press

Garson JW (2001) Quantification in modal logic. In: Gabbay DM, Guenthner F (eds) Handbook of Philosophical Logic, Springer, Dordrecht, pp 267-323

Gattinger M, van Eijck J, Wang Y (2017) Knowing values and public inspection. In: Proceedings of ICLA' 17, forthcoming

Gochet P (2013) An open problem in the logic of knowing how. In: Hintikka J (ed) Open Problems in Epistemology, The Philosophical Society of Finland 
Gochet P, Gribomont P (2006) Epistemic logic. In: Gabbay DM, Woods J (eds) Handbook of the History of Logic, vol 7

Goranko V, Kuusisto A (2015) Logics for propositional determinacy and independence, URL https://arxiv.org/abs/1609.07398, manuscript

Gu T, Wang Y (2016) "knowing value" logic as a normal modal logic. In: Proceedings of AiML Vol.11, 362-381

Harrah D (2002) The logic of questions. In: Gabbay D (ed) Handbook of Philosophical Logic, vol 8

Hart S, Heifetz A, Samet D (1996) "knowing whether", "knowing that", and the cardinality of state spaces. Journal of Economic Theory 70(1):249-256

Heim I (1979) Concealed questions. In: Bäuerle R, Egli U, von Stechow A (eds) Semantics from Different Points of View, pp 51-60

Herzig A (2015) Logics of knowledge and action: critical analysis and challenges. Autonomous Agents and Multi-Agent Systems 29(5):719-753

Herzig A, Lorini E, Maffre F (2015) A poor man's epistemic logic based on propositional assignment and higher-order observation. In: Proceedings of LORI-V, pp $156-168$

Hintikka J (1962) Knowledge and Belief: An Introduction to the Logic of the Two Notions. Cornell University Press, Ithaca N.Y.

Hintikka J (1989a) On sense, reference, and the objects of knowledge. In: The Logic of Epistemology and the Epistemology of Logic: Selected Essays, Springer, pp $45-61$

Hintikka J (1989b) Reasoning about knowledge in philosophy: The paradigm of epistemic logic. In: The Logic of Epistemology and the Epistemology of Logic: Selected Essays, Springer, pp 17-35

Hintikka J (1996) Knowledge acknowledged: Knowledge of propositions vs. knowledge of objects. Philosophy and Phenomenological Research 56(2):251-275

Hintikka J (1999) What is the logic of experimental inquiry? In: Inquiry as Inquiry: A Logic of Scientific Discovery, Springer, pp 143-160

Hintikka J (2003) A second generation epistemic logic and its general significance. In: Hendricks VF, Jørgensen KF, Pedersen SA (eds) Knowledge Contributors, Springer, pp 33-55

Hintikka J (2007) Socratic epistemology: Explorations of knowledge-seeking by questioning. Cambridge: Cambridge University Press

Hintikka J, Halonen I (1995) Semantics and pragmatics for why-questions. The Journal of Philosophy 92(12):636-657

Hintikka J, Sandu G (1989) Informational independence as a semantical phenomenon. In: Fenstad JE, Frolov IT, Hilpinen R (eds) Logic, Methodology and Philosophy of Science 8, Elsevier, pp 571-589

Hintikka J, Symons J (2003) Systems of visual identification in neuroscience: Lessons from epistemic logic. Philosophy of Science 70(1):89-104

Hodkinson IM (2002) Monodic packed fragment with equality is decidable. Studia Logica 72(2):185-197

Hodkinson IM, Wolter F, Zakharyaschev M (2000) Decidable fragment of first-order temporal logics. Annals of Pure and Applied Logic 106(1-3):85-134 
Hodkinson IM, Wolter F, Zakharyaschev M (2002) Decidable and undecidable fragments of first-order branching temporal logics. In: Proceedings of LiCS'02, pp 393-402

van der Hoek W, Lomuscio A (2004) A logic for ignorance. Electronic Notes in Theoretical Computer Science 85(2):117-133

Holliday WH, Perry J (2014) Roles, rigidity, and quantification in epistemic logic. In: Baltag A, Smets S (eds) Johan van Benthem on Logic and Information Dynamics, Springer, pp 591-629

Humberstone L (1995) The logic of non-contingency. Notre Dame Journal of Formal Logic 36(2):214-229

Kaneko M, Nagashima T (1996) Game logic and its applications. Studia Logica 57(2/3):325-354

Khan MA, Banerjee M (2010) A logic for multiple-source approximation systems with distributed knowledge base. Journal of Philosophical Logic 40(5):663-692

Kuhn S (1995) Minimal non-contingency logic. Notre Dame Journal of Formal Logic 36(2):230-234

Lau T, Wang Y (2016) Knowing your ability. The Philosophical Forum pp 415-424

Lenzen W (1978) Recent work in epistemic logic. Acta Philosophica Fennica $30(2): 1-219$

Li Y, Wang Y (2017) Achieving while maintaining: A logic of knowing how with intermediate constraints. In: Proceedings of ICLA'17, forthcoming

Liu F, Wang Y (2013) Reasoning about agent types and the hardest logic puzzle ever. Minds and Machines 23(1):123-161

Lomuscio A, Ryan M (1999) A spectrum of modes of knowledge sharing between agents. In: Proceedings of ATAL'99, pp 13-26

Ma X, Guo W (1983) W-JS: A modal logic of knowledge. In: Proceedings of the 8th International Joint Conference on Artificial Intelligence. Karlsruhe, FRG, August 1983, pp 398-401

McCarthy J (1979) First-Order theories of individual concepts and propositions. Machine Intelligence 9.:129-147

Montgomery H, Routley R (1966) Contingency and non-contingency bases for normal modal logics. Logique et Analyse 9:318-328

Moore RC (1977) Reasoning about knowledge and action. In: Proceedings of IJCAI'77, pp 223-227

Petrick RPA, Bacchus F (2004a) Extending the knowledge-based approach to planning with incomplete information and sensing. In: Zilberstein S, Koehler J, Koenig S (eds) Proceedings of ICAPS'04), AAAI Press, pp 2-11

Petrick RPA, Bacchus F (2004b) PKS: Knowledge-based planning with incomplete information and sensing. In: Proceedings of ICAPS'04

Pizzi C (2007) Necessity and relative contingency. Studia Logica 85(3):395-410

Plaza JA (1989) Logics of public communications. In: Emrich ML, Pfeifer MS, Hadzikadic M, Ras ZW (eds) Proceedings of the 4th International Symposium on Methodologies for Intelligent Systems, pp 201-216

Ryle G (1949) The Concept of Mind. Penguin

Stanley J (2011) Know how. Oxford University Press 
Stanley J, Williamson T (2001) Knowing how. Journal of Philosophy 98:411-444

Sturm H, Wolter F, Zakharyaschev M (2000) Monodic epistemic predicate logic. In: Proceedings of JELIA'00, pp 329-344

Väänänen J (2007) Dependence Logic: A New Approach to Independence Friendly Logic. Cambridge University Press

Van Ditmarsch H, Herzig A, De Lima T (2011) From situation calculus to dynamic epistemic logic. Journal of Logic and Computation pp 179-204

Von Wright GH (1951) An Essay in Modal Logic. North Holland, Amsterdam

Wang Y (2015a) A logic of knowing how. In: Proceedings of LORI-V, pp 392-405

Wang Y (2015b) Representing imperfect information of procedures with hyper models. In: Proceedings of ICLA'15, pp 218-231

Wang Y (2017) A logic of goal-directed knowing how. Synthese Forthcoming

Wang Y, Fan J (2013) Knowing that, knowing what, and public communication: Public announcement logic with Kv operators. In: Proceedings of IJCAI'13, pp $1139-1146$

Wang Y, Fan J (2014) Conditionally knowing what. In: Proceedings of AiML Vol.10, pp 569-587

Wolter F (2000) First order common knowledge logics. Studia Logica 65(2):249_ 271

Xiong S (2014) Decidability of $\mathbf{E} \mathbf{L} \mathbf{K} \mathbf{v}^{\mathbf{r}}$. Bachelor's thesis, Peking University (in Chinese)

Xu C, Wang Y, Studer T (2016) A logic of knowing why, under submission

Yu Q, Li Y, Wang Y (2016) A dynamic epistemic framework for conformant planning. In: Proceedings of TARK'15, EPTCS, forthcoming

Zolin E (1999) Completeness and definability in the logic of noncontingency. Notre Dame Journal of Formal Logic 40(4):533-547

Zolin E (2001) Infinitary expressibility of necessity in terms of contingency. In: Striegnitz K (ed) Proceedings of the Sixth ESSLLI Student Session, pp 325-334 